\title{
Broadly neutralizing antibody cocktails targeting Nipah virus and Hendra virus fusion glycoproteins
}

\author{
Ha V. Dang ${ }^{1}$, Robert W. Cross ${ }^{2,3}$, Viktoriya Borisevich², Zachary A. Bornholdt ${ }^{4}$, Brandyn R. West ${ }^{4}$, \\ Yee-Peng Chan ${ }^{5}$, Chad E. Mire ${ }^{2,3}$, Sofia Cheliout Da Silva5, Antony S. Dimitrov ${ }^{5}$, Lianying Yan ${ }^{5}$, \\ Moushimi Amaya5 , Chanakha K. Navaratnarajah6 ${ }^{6}$, Larry Zeitlin $\mathbb{1}^{4}$, Thomas W. Geisbert ${ }^{2,3}$, \\ Christopher C. Broder $\mathbb{D}^{5}$ and David Veesler ${ }^{1 凶}$
}

\begin{abstract}
Hendra virus (HeV) and Nipah virus (NiV) are henipaviruses (HNVs) causing respiratory illness and severe encephalitis in humans, with fatality rates of 50-100\%. There are no licensed therapeutics or vaccines to protect humans. $\mathrm{HeV}$ and $\mathrm{NiV}$ use a receptor-binding glycoprotein (G) and a fusion glycoprotein (F) to enter host cells. HNV F and $G$ are the main targets of the humoral immune response, and the presence of neutralizing antibodies is a correlate of protection against NiV and $\mathrm{HeV}$ in experimentally infected animals. We describe here two cross-reactive F-specific antibodies, 1F5 and 12B2, that neutralize NiV and $\mathrm{HeV}$ through inhibition of membrane fusion. Cryo-electron microscopy structures reveal that 1F5 and $12 \mathrm{~B} 2$ recognize distinct prefusion-specific, conserved quaternary epitopes and lock $F$ in its prefusion conformation. We provide proof-of-concept for using antibody cocktails for neutralizing $\mathrm{NiV}$ and $\mathrm{HeV}$ and define a roadmap for developing effective countermeasures against these highly pathogenic viruses.
\end{abstract}

$\mathrm{N}$ ipah virus $(\mathrm{NiV})$ and Hendra virus $(\mathrm{HeV})$ are zoonotic pathogens of the Henipavirus (HNV) genus causing encephalitis and respiratory symptoms in humans with fatality rates of $50-100 \%$ (ref. ${ }^{1}$ ). HNVs have an exceptionally broad species tropism infecting animals spanning at least six mammalian orders ${ }^{1}$. Transmission to humans can occur directly from Pteropid fruit bats (the reservoir host), pigs, horses or from humans ${ }^{2-5}$. Multiple cross-species transmissions occurred in Australia and South/ Southeast Asia since the discovery of $\mathrm{HeV}$ and $\mathrm{NiV}$ in the mid to late 1990s. Furthermore, the recent detection of cross-reactive HNVs antibodies (Abs) in humans and Pteropus bats in Africa ${ }^{6}$ underscored that 2 billion people worldwide live in regions threatened by HNV spillover events. So far, no approved vaccines or therapeutics exist to protect humans against $\mathrm{HNV}$ infections.

HNVs are members of the paramyxovirus family, which includes a number of pathogens causing disease in humans such as measles, mumps and parainfluenza viruses. Paramyxovirus entry into host cells requires fusion of the viral membrane and the host plasma membrane. This process is mediated by the interplay between two glycoproteins present at the viral surface: an attachment protein $(\mathrm{H}$, $\mathrm{HN}$ or G, depending on the presence of hemagglutinin and/or neuraminidase activities) and a fusion protein $(\mathrm{F})^{7}$. These two glycoproteins are known as $\mathrm{G}$ and $\mathrm{F}$ for $\mathrm{NiV}, \mathrm{HeV}$ and other HNVs. HNV $\mathrm{G}$ is a homotetrameric type II transmembrane protein with a large ectodomojmain comprising an $\mathrm{N}$-terminal stalk and a C-terminal beta-propeller receptor-binding domain ${ }^{8,9}$. The $\mathrm{NiV}$ and $\mathrm{HeV}$ entry receptors at the surface of host cells are the transmembrane protein tyrosine kinases ephrin-B2 or ephrin-B3 (refs. ${ }^{10-13}$ ). HNV F is a trimeric type I transmembrane protein synthesized as an $F_{0}$ precursor that is proteolytically cleaved by host cathepsin $\mathrm{L}$ to yield the fusion-active, disulfide-linked $\mathrm{F}_{2} / \mathrm{F}_{1}$ subunits ${ }^{14,15}$. The $\mathrm{N}$ terminus comprises the $\mathrm{F}_{2}$ subunit whereas the $\mathrm{C}$-terminal $\mathrm{F}_{1}$ subunit contains a hydrophobic fusion peptide and two heptad repeat regions, designated HRA and $\mathrm{HRB}^{16,17}$. Both $\mathrm{G}$ and $\mathrm{F}$ are required for $\mathrm{HNV}$ infection and they have been proposed to undergo a cascade of conformational changes to promote virus-host membrane fusion on receptor engagement ${ }^{18-23}$.

As for many other enveloped viruses, the HNV glycoproteins are the only viral antigens displayed on the surface of the virion and are therefore the main targets of virus-neutralizing Abs. Moreover, the presence of neutralizing Abs is a correlate of protection against $\mathrm{NiV}$ and $\mathrm{HeV}$ in experimentally infected animals $\mathrm{s}^{24-26}$. An $\mathrm{Ab}$ targeting the $\mathrm{G}$ glycoprotein and preventing receptor engagement ${ }^{27-29}$, m102.4, was previously used on a compassionate basis to treat individuals with serious $\mathrm{HeV}$ or $\mathrm{NiV}$ exposure risk in Australia and the USA, and successfully completed a phase I safety trial in Australia, which will guide its future use under emergency setting ${ }^{30}$. We previously characterized an $\mathrm{Ab}$ designated $5 \mathrm{~B} 3$ that cross-reacts with the $\mathrm{NiV}$ and $\mathrm{HeV}$ F glycoproteins, potently inhibits infections of authentic $\mathrm{NiV}$ and $\mathrm{HeV}$ and protects ferrets when administered up to several days postinfection ${ }^{31,32}$. While these results paved the way for using m102.4 or h5B3.1 (the humanized version of 5B3) in either a prophylactic or therapeutic capacity, a comprehensive understanding of $\mathrm{F}$ and $\mathrm{G}$ glycoprotein epitopes targeted by neutralizing Abs remains lacking. Detailed epitope information is required to design effective strategies to combat $\mathrm{NiV}$ and $\mathrm{HeV}$ as well as other putatively emerging HNVs, such as Ghana virus $(\mathrm{GhV})^{6,33}$ or Mojiang virus ${ }^{34}$.

We describe here two mouse monoclonal Abs, 12B2 and 1F5, along with humanized variants, designated h12B2 and h1F5, recognizing the $\mathrm{NiV} \mathrm{F}$ and $\mathrm{HeV}$ F glycoproteins. We demonstrate $12 \mathrm{~B} 2$ and 1F5 bind with high affinity to NiV F and $\mathrm{HeV} F$ and neutralize

'Department of Biochemistry, University of Washington, Seattle, WA, USA. ${ }^{2}$ Department of Microbiology and Immunology, University of Texas Medical Branch, Galveston, TX, USA. ${ }^{3}$ Galveston National Laboratory, University of Texas Medical Branch, Galveston, TX, USA. ${ }^{4}$ Mapp Biopharmaceutical, Inc., San Diego, CA, USA. ${ }^{5}$ Department of Microbiology and Immunology, Uniformed Services University, Bethesda, MD, USA. ${ }^{6}$ Department of Molecular Medicine, Mayo Clinic, Rochester, MN, USA. $₫ e$-mail: dveesler@uw.edu 
authentic $\mathrm{NiV}$ and $\mathrm{HeV}$ under biosafety level 4 (BSL-4) containment. We determined cryogenic-electron microscopy (cryo-EM) structures of NiV F in complex with $12 \mathrm{~B} 2$ and of $\mathrm{HeV} F$ in complex with 1F5 at 2.9 and $2.8 \AA$ resolutions, respectively, and show the two Abs recognize distinct, prefusion-specific, quaternary epitopes that are conserved in $\mathrm{NiV}$ and $\mathrm{HeV}$ F. Our structural data combined with membrane fusion assays indicate that $12 \mathrm{~B} 2$ and 1F5 stabilize $\mathrm{F}$ in the prefusion state, which is the conformation found at the viral surface before infection, and prevent the structural rearrangements leading to membrane fusion. We delineate two key $\mathrm{F}$ regions targeted by cross-reactive Abs neutralizing $\mathrm{NiV}$ and $\mathrm{HeV}$, either in isolation or formulated as cocktails, and define a path forward for developing $\mathrm{Ab}$ cocktails that reduce the propensity to elicit viral escape mutants compared to single Ab treatments.

\section{Results}

12B2 and 1F5 broadly neutralize $\mathrm{NiV}$ and $\mathrm{HeV}$ by blocking membrane fusion. To explore the humoral immune response against the HNV F glycoprotein, we sequenced the variable regions of the 1F5 and 12B2 Abs from hybridomas isolated from mice immunized with a prefusion stabilized $\mathrm{NiV} \mathrm{F}$ ectodomain trimer $^{18}$. We used biolayer interferometry (BLI) to characterize binding of the 12B2 and 1F5 Fab fragments to prefusion $\mathrm{NiV} F$ or $\mathrm{HeV}$ F ectodomain trimers immobilized on the surface of biosensors (Fig. 1a-d and Supplementary Table 1). We determined equilibrium dissociation constants $\left(K_{\mathrm{d}}\right)$ of 27.7 and $5.35 \mathrm{nM}$ for attachment of 12B2 to NiV $\mathrm{F}$ or $\mathrm{HeV} \mathrm{F}$, respectively. For $1 \mathrm{~F} 5$ recognition of $\mathrm{NiV} \mathrm{F}$ and $\mathrm{HeV} \mathrm{F}$, we obtained respective equilibrium dissociation constants of 1.47 and $1.24 \mathrm{nM}$, indicating this Fab bound more tightly than $12 \mathrm{~B} 2$ to the two tested HNV F glycoproteins. These data establish that each of the two Abs cross-react with $\mathrm{NiV} \mathrm{F}$ and $\mathrm{HeV} \mathrm{F}$ in their prefusion conformation (Fig. 1a-d and Supplementary Table 1).

To evaluate the effect of 12B2 or 1F5 on membrane fusion, we used a split-luciferase-reporter cell-cell fusion assay with effector and target cells expressing HNV G/F or ephrin-B2, respectively ${ }^{35,36}$. We observed that 12B2, 1F5 and the previously described 5B3 (ref. ${ }^{31}$ ) inhibited $\mathrm{NiV}$ and $\mathrm{HeV} \mathrm{F} / \mathrm{G}$-mediated membrane fusion in a concentration-dependent manner and that 1F5 outperformed the other two Abs in this assay (Fig. 1e,f).

We next humanized 1F5 (h1F5) and 12B2 (h12B2) and evaluated the neutralization potency and breadth of these Abs against authentic NiV-Malaysia (NiV-M), NiV-Bangladesh (NiV-B) and $\mathrm{HeV}$. Mean half-maximal inhibitory concentrations $\left(\mathrm{IC}_{50}\right)$ were determined using plaque reduction assays to monitor viral neutralization in the presence of increasing $\mathrm{Ab}$ concentrations (Fig. 1g-i and Supplementary Table 2). We found that our Abs broadly inhibited $\mathrm{NiV}-\mathrm{M}, \mathrm{NiV}-\mathrm{B}$ and $\mathrm{HeV}$ with $\mathrm{IC}_{50}$ ranging between 0.2 and $1.3 \mu \mathrm{g} \mathrm{ml}^{-1}$ for $1 \mathrm{~F} 5-\mathrm{h} 1 \mathrm{~F} 5$ and $0.4-3.6 \mu \mathrm{g} \mathrm{ml}^{-1}$ for $12 \mathrm{~B} 2-\mathrm{h} 12 \mathrm{~B} 2$. Furthermore, the humanized versions of $12 \mathrm{~B} 2$ and $1 \mathrm{~F} 5$ retained the neutralization potency and breadth of the parental Abs.

Structure of NiV F in complex with the 12B2 neutralizing Ab. To study the mechanism of 12B2-mediated neutralization of $\mathrm{NiV}$ and $\mathrm{HeV}$, we determined a cryo-EM structure of the NiV F ectodomain trimer in complex with the 12B2 Fab fragment at $2.9 \AA$ resolution (Fig. $2 \mathrm{a}-\mathrm{c}$, Supplementary Fig. 1 and Table 1). The local resolution is highest for most of NiV F and the Fab variable domains whereas the (peripheral) constant domains are less well-resolved and were not modeled in density. The final model includes NiV F residues 27-481 with a chain break at the boundary between the $F_{2}$ and $F_{1}$ subunits (residues 105-111 were not resolved).

12B2 binds to a previously undescribed quaternary epitope in domain III near the $\mathrm{F}$ apex (that is, distal to the viral membrane) with three Fabs bound to an $\mathrm{F}$ trimer. The Fab complementary-determining regions (CDRs) CDRH1-H3, CDRL1-L3 and framework regions (FR) FRL1, FRH1 and FRH3 contribute to burying roughly $1,400 \AA^{2}$ at the F interface via electrostatic interactions and shape complementarity (Fig. 2d,e). The paratope is dominated by the 12B2 heavy chain (Fig. 2d) that engages two adjacent $\mathrm{F}$ protomers whereas the Fab light chain contacts a single protomer. 12B2 simultaneously sits atop the HRA $\beta$-hairpin of one $\mathrm{F}$ protomer and the HRA/central helix region of the neighboring protomer, contacting both the $F_{2}$ and $F_{1}$ subunits via involvement of the upstream helix-preceding region of both protomers.

CDRH1-H3 and FRH3 recognize HRA through a constellation of interactions between (1) the CDRH1 Ser28 $8_{12 \mathrm{~B} 2}$ and Ser $153_{\mathrm{NiV}}$ side chains; (2) the CDRH1 Ser31 $1_{12 \mathrm{~B} 2}$ main chain carbonyl and side chain hydroxyl with the Asn $155_{\mathrm{NiV}}$ amide side chain and the Ser $153_{\mathrm{NiV}}$ backbone carbonyl, respectively; (3) the CDRH2 Thr53 $3_{12 \mathrm{~B} 2}$ and the Glu156 $6_{\mathrm{NiV}}$ side chains; (4) the backbone carbonyl of CDRH2 Gly5 $4_{12 \mathrm{~B} 2}$ and the backbone amide group of Lys $160_{\mathrm{Niv}}$; (5) the FRH3 Asn $70_{12 \mathrm{~B} 2}$ and Gln162 $2_{\mathrm{NiV}}$ side chains; (6) the Arg71 ${ }_{12 \mathrm{~B} 2}$ backbone carbonyl and side chain guanidinium with the Gln $162_{\mathrm{NiV}}$ side chain and Lys $160_{\mathrm{NiV}}$ backbone carbonyl, respectively; (7) the FRH3 Asn73 ${ }_{12 \mathrm{~B} 2}$ backbone and side chain amides as well as backbone carbonyl with the Gln $162_{\mathrm{NiV}}$ backbone carbonyl and amide and Ser $150_{\mathrm{Niv}}$ hydroxyl, respectively, and (8) the CDRH3 Tyr101 ${ }_{12 \mathrm{~B} 2}$ and Asn67 $7_{\mathrm{NiV}}$ side chains. The HRA $\beta$-hairpin adopts a conformation similar to the apo $\mathrm{HeV}$ $\mathrm{F}^{16}$ and $\mathrm{NiV} \mathrm{F}^{1738}$ structures. 12B2 engagement of the neighboring protomer involves hydrogen bonding between the CDRH2 Ser56 $12 \mathrm{~B} 2$ hydroxyl and Lys $193_{\mathrm{Niv}}$ amine side chains as well as the CDRH3 Tyr $101_{12 \mathrm{~B} 2}$ backbone carbonyl and Ser $191_{\mathrm{NiV}}$ side chain hydroxyl in addition to the CRDL1 Tyr32 $2_{12 \mathrm{~B} 2}$ side chain hydroxyl-mediated contacts with the Ile187 $\mathrm{NiV}$ and Ile190 ${ }_{\mathrm{NiV}}$ backbone carbonyl in the $\mathrm{HRA}$ /central helix region. $12 \mathrm{~B} 2$ binding induces a $90^{\circ}$ rotation of the Asn67 $7_{\mathrm{NiV}} \mathrm{N}$-linked glycan, which is moved out of the way to grant the Fab access to the proteinaceous part of the NiV F epitope (Fig. 2f). This structural change is stabilized via multiple electrostatic contacts between the oligosaccharide and the heavy chain $G \ln 1_{12 \mathrm{~B} 2}$, CDRH1, CDRH 3 and CDRL2, which contribute $18 \%$ of the surface buried by the paratope at the interface with $\mathrm{NiV} \mathrm{F}$.

To evaluate the impact of the oligosaccharide at position Asn67 on $12 \mathrm{~B} 2$ recognition, we generated the S69A NiV F point mutant in which the $\mathrm{N}$-glycosylation sequon is abolished. BLI analysis of 12B2 Fab fragment binding to NiV F S69A glycan mutant revealed a markedly higher affinity compared to wildtype $\mathrm{NiV} \mathrm{F}$, which was virtually exclusively achieved through a tenfold on-rate enhancement (Supplementary Fig. 2 and Supplementary Table 1). These results therefore indicate that although the N67 glycan dampens antibody recognition, 12B2 overcomes this steric barrier by making favorable interactions with the N67 glycan to bind to the NiV and $\mathrm{HeV} \mathrm{F}$ trimers.

Analysis of the 12B2 epitope conservation between $\mathrm{NiV} \mathrm{F}$ and $\mathrm{HeV} F$ shows that virtually all residues buried by $12 \mathrm{~B} 2$ binding are strictly conserved, including the oligosaccharide at position Asn67 that directly participates in binding (Fig. $2 \mathrm{~g}$ ). The $\mathrm{M}_{\mathrm{Niv}} 68 \mathrm{~V}_{\mathrm{HeV}}$ substitution is not expected to affect $\mathrm{Ab}$ recognition, as both side chains point toward the $\mathrm{F}$ core. The $\mathrm{Q}_{\mathrm{NiV}} 70 \mathrm{~K}_{\mathrm{HeV}}$ substitution might enhance interaction with $\mathrm{HeV}$ (putatively through contacts with CDRL1 E27) whereas the $\mathrm{K}_{\mathrm{Niv}} 189 \mathrm{Q}_{\mathrm{HeV}}$ substitution would not have a major impact on binding as this side chain is not involved in specific interactions with 12B2. The structural data therefore rationalize the roughly comparable affinities measured for binding of the 12B2 Fab fragment to NiV F and HeV F (Fig. 1a-d and Supplementary Table 1). These results demonstrate that the epitopes recognized by potent HNV F-targeting Abs can involve oligosaccharides, as has been described for some SARS-CoV-2 (refs. ${ }^{37,38}$ ), MERS-CoV ${ }^{39}$, HIV-1 (refs. ${ }^{40,41}$ ) and $\mathrm{EBOV}^{42}$ Abs.

Structure of $\mathrm{HeV}$ F in complex with the 1F5 neutralizing Ab. To understand the 1F5-mediated neutralization of $\mathrm{NiV}$ and $\mathrm{HeV}$, we determined a cryo-EM structure of the $\mathrm{HeV} F$ ectodomain 
a

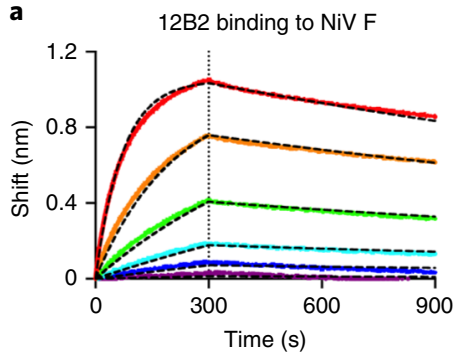

c

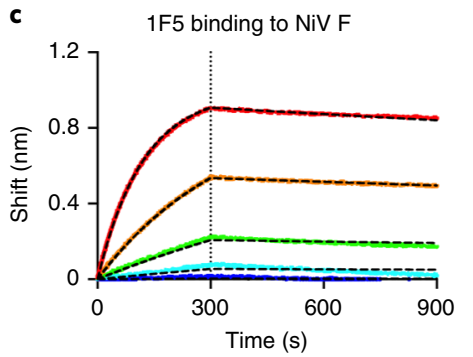

e

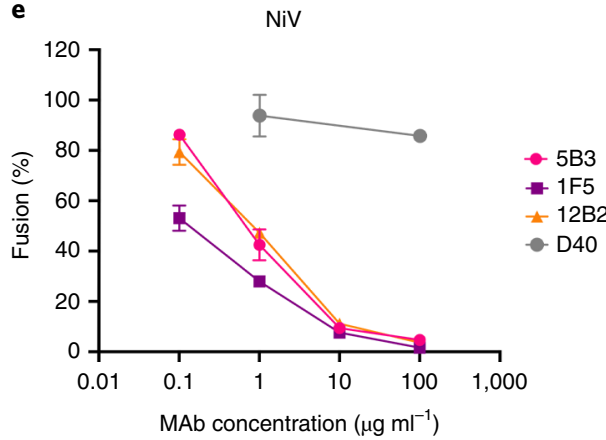

b
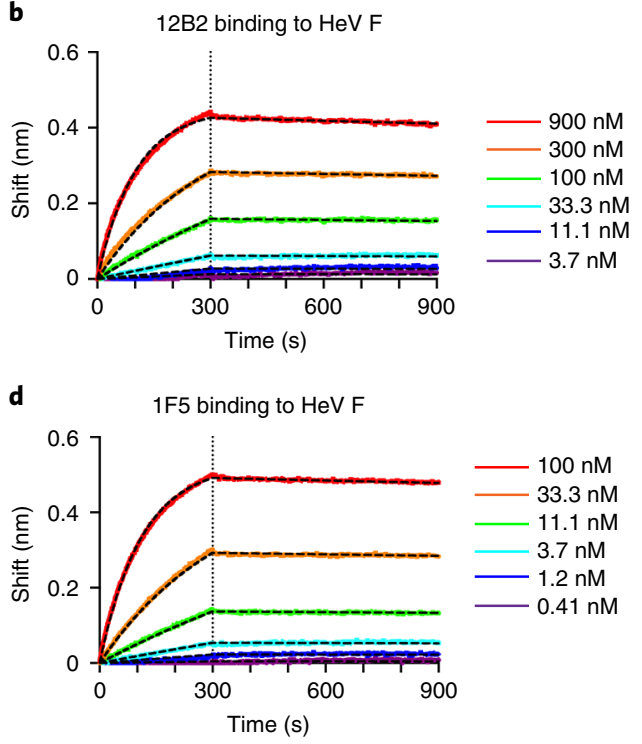

f

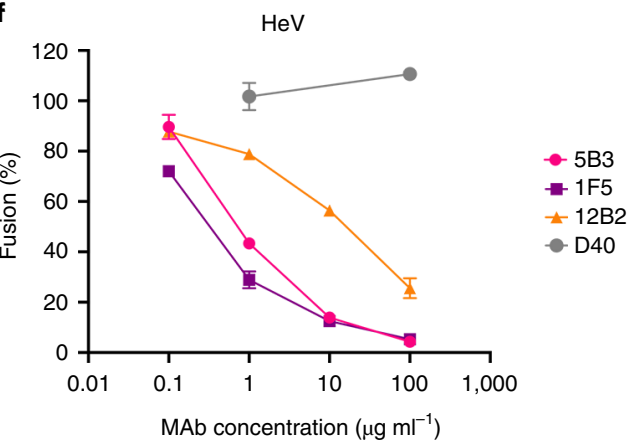

g

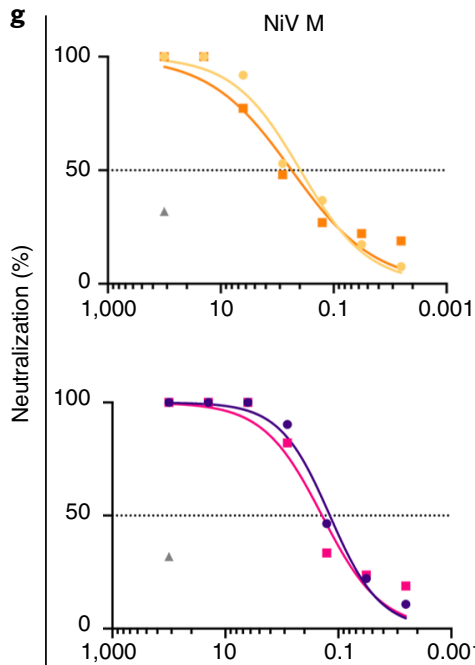

h
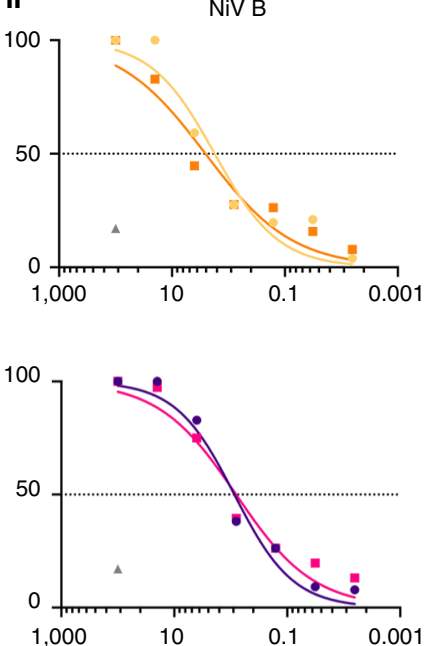
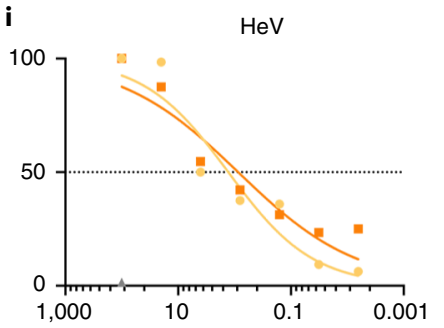

12B2

h12B2

$\triangle \mathrm{C} 13 \mathrm{G} 8$

MAb concentration $\left(\mu \mathrm{g} \mathrm{ml}^{-1}\right)$

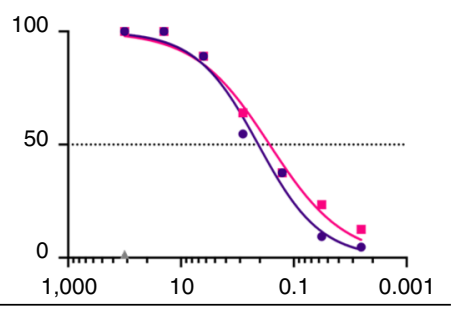

.001

Fig. 1 | The 12B2 and $1 F 5$ Abs cross-react with NiV F and HeV F and neutralize authentic NiV and $\mathrm{HeV}$ by preventing membrane fusion. a-d, Binding of 12B2 (a,b) or $1 F 5(\mathbf{c}, \mathbf{d})$ Fab fragments to immobilized NiV F (a,c) or HeV F (b,d) ectodomains analyzed by BLI. Raw data are colored according to the key, and fitted curves are displayed as black dashed lines. The vertical dotted lines correspond to the transition between the association and dissociation phases. The experiments were performed in replicates with two different preparations of NiV F and of HeV F. A representative experiment is shown. e,f, $1 \mathrm{~F} 5$ and 12B2 inhibit membrane fusion mediated by $\mathrm{NiV}(\mathbf{e})$ and $\mathrm{HeV}(\mathbf{f})$ glycoproteins in a fusion inhibition assay with split luciferase. D40 is a mAb against HIV gp-41 used as a negative control ${ }^{52}$. g-i, Neutralization of authentic NiV-M (g), NiV-B (h) and HeV (i) by 12 B2 and h12B2 (top row) and 1F5 and h1F5 (bottom row) Abs. c13G8 (gray) is an anti-Crimean-Congo hemorrhagic fever virus mAb used as a negative control. Data shown are from one replicate of two independent experiments with independent virus preparations. $I C_{50}$ values for each replicate of the experiment are reported in Supplementary Table 2. 
$\mathbf{a}$

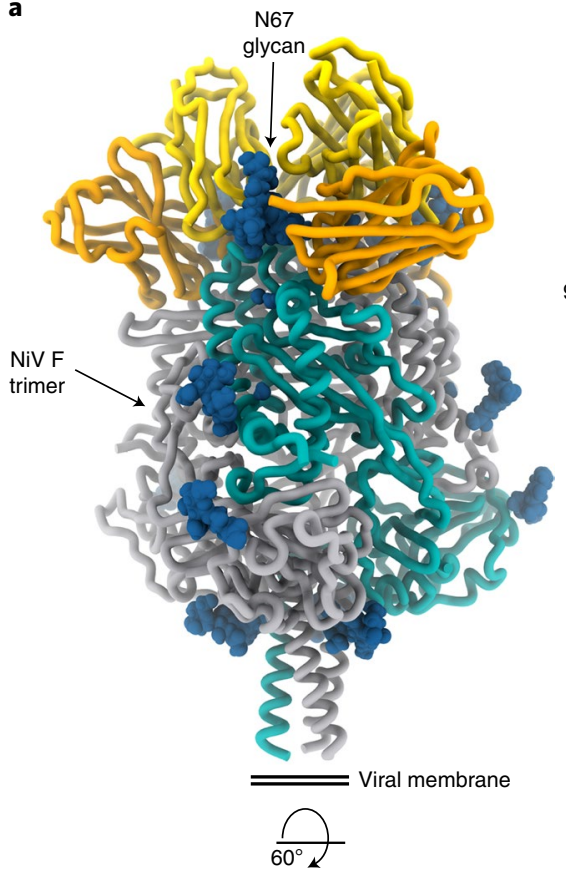

c

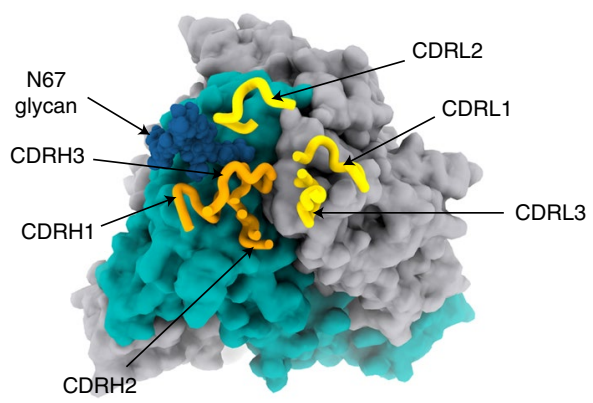

f

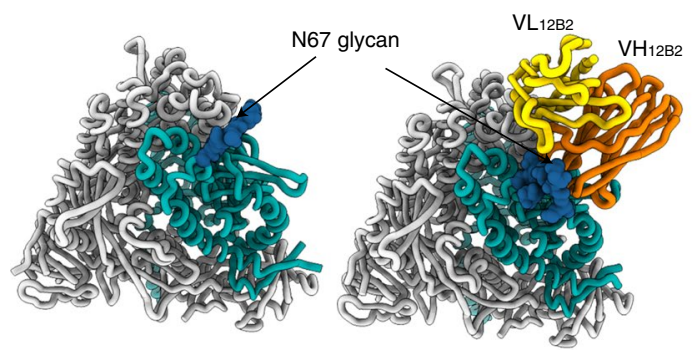

b

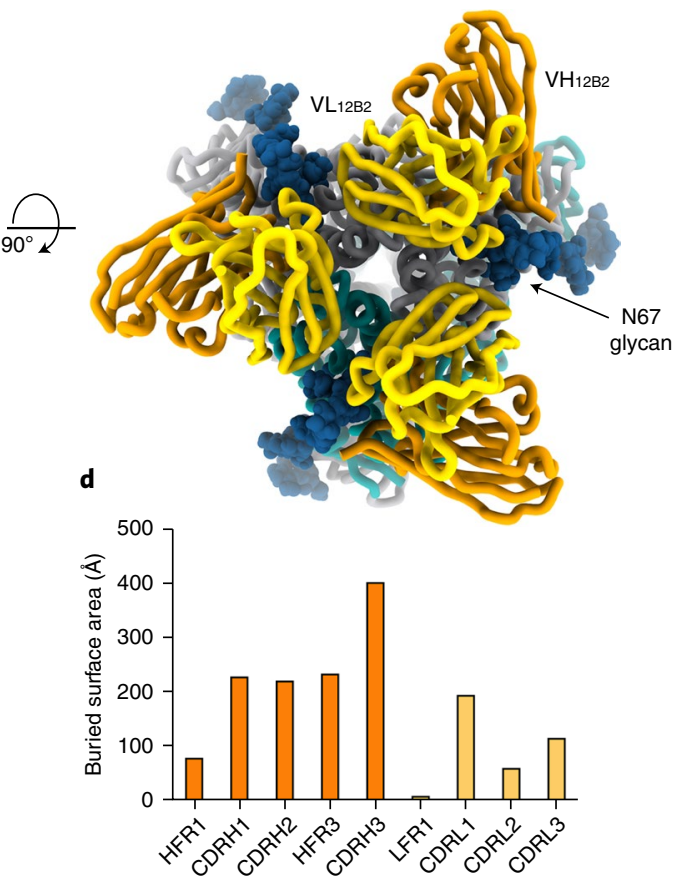

e

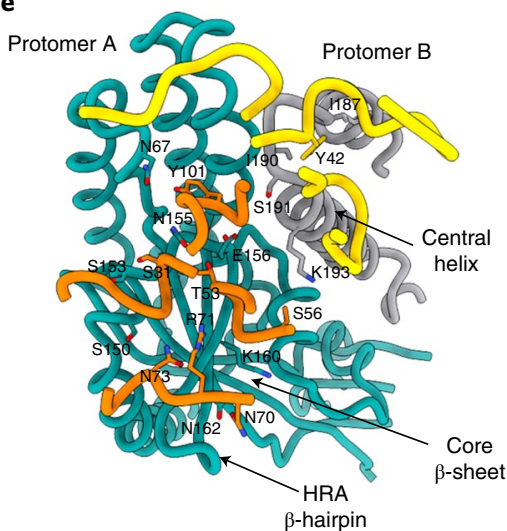

g

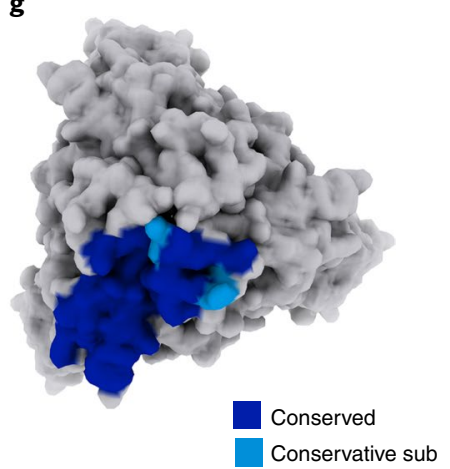

Fig. 2 | The 12B2 Ab recognizes a conserved quaternary epitope on the NiV F glycoprotein. a,b, Ribbon diagrams showing two orthogonal views (a, side view; $\mathbf{b}$, top view) of the cryo-EM structure of the NiV F ectodomain trimer in complex with the 12B2 Fab fragment. One F protomer is rendered in teal and the other two protomers in gray. c, Molecular surface representation of the NiV F trimer with the CDR loops of a single 12B2 Fab shown as ribbons, highlighting the quaternary nature of the epitope. $\mathbf{d}$, Contribution of distinct regions of 12B2 to epitope recognition at the surface of NiV F. CDRH, heavy chain CDR; CDRL, light chain CDR; HFR, heavy chain framework region; LFR, light chain framework region. e, Zoomed-in view of the interface between NiV $\mathrm{F}$ and 12B2 with selected residues rendered as sticks. The NiV F glycan at position Asn67 is not shown. One protomer of NiV F is colored in teal and an adjacent protomer in gray. In a-e, the 12B2 variable heavy (VH12B2) and light (VL12B2) chains are colored orange and gold, respectively. f, Molecular surface representation of the NiV F trimer showing the 12B2 footprint colored by residue conservation between NiV F and HeV F glycoproteins. Conservative sub, conservative substitution. $\mathbf{g}$, Comparison of NiV F without (left, PDB 6TYS) and with bound 12B2 Fab (right) showing the rotation of NiV F glycan Asn67. 
Table 1 | Cryo-EM data collection, refinement and validation statistics

NiV F-12B2 (EMD- HeV F-1F5 (EMD22884, PDB 7KI4) 22885, PDB 7KI6)

\begin{tabular}{|c|c|c|}
\hline \multicolumn{3}{|l|}{ Data collection and processing } \\
\hline Magnification (nominal) & 130,000 & 130,000 \\
\hline Voltage (kV) & 300 & 300 \\
\hline Electron exposure $\left(\mathrm{e}^{-} / \AA^{2}\right)$ & 70 & 70 \\
\hline Defocus range $(\mu \mathrm{m})$ & $0.9-3.5$ & $0.6-3.2$ \\
\hline Pixel size $(\AA)$ & 1.05 & 1.05 \\
\hline Symmetry imposed & C3 & C3 \\
\hline Initial particle images (no.) & 525,376 & 432,222 \\
\hline Final particle images (no.) & 28,776 & 34,415 \\
\hline Map resolution $(\AA)$ & 2.9 & 2.8 \\
\hline FSC threshold & 0.143 & 0.143 \\
\hline \multicolumn{3}{|l|}{ Refinement } \\
\hline Initial model used (PDB ID) & PDB 6TYS & PDB 5EJB \\
\hline Model resolution $(\AA)$ & 3.2 & 2.9 \\
\hline FSC threshold & 0.5 & 0.5 \\
\hline Map sharpening $B$ factor $\left(\AA^{2}\right)$ & -51 & -52 \\
\hline \multicolumn{3}{|l|}{ Model composition } \\
\hline Protein residues & 2,010 & 1,986 \\
\hline \multicolumn{3}{|l|}{$B$ factors $\left(\AA^{2}\right)$} \\
\hline Protein & 17.6 & 12.13 \\
\hline \multicolumn{3}{|l|}{ R.m.s. deviations } \\
\hline Bond lengths $(\AA)$ & 0.012 & 0.013 \\
\hline Bond angles $\left({ }^{\circ}\right)$ & 1.26 & 1.31 \\
\hline \multicolumn{3}{|l|}{ Validation } \\
\hline MolProbity score & 1.00 & 1.07 \\
\hline Clashscore & 1.35 & 2.83 \\
\hline Poor rotamers (\%) & 0 & 0.17 \\
\hline \multicolumn{3}{|l|}{ Ramachandran plot } \\
\hline Favored (\%) & 97.28 & 98.78 \\
\hline Allowed (\%) & 2.57 & 1.22 \\
\hline Disallowed (\%) & 0.15 & 0.00 \\
\hline EMRinger score & 4.04 & 4.32 \\
\hline
\end{tabular}

trimer in complex with the 1F5 Fab fragment at $2.8 \AA$ resolution (Fig. $3 a-c$, Supplementary Fig. 3 and Table 1). As was the case for the NiV F-12B2 and NiV F-5B3 structures, the map is best resolved for most of $\mathrm{HeV} \mathrm{F}$ and the Fab variable domains whereas the peripheral Fab constant domains are weakly resolved and were not modeled. The final model includes $\mathrm{HeV}$ F residues 26-471 with a chain break at the boundary between the $\mathrm{F}_{2}$ and $\mathrm{F}_{1}$ subunits (residues 104-112 were not resolved).

1F5 binds to a glycan-free quaternary epitope on the side of domain III of the HeV F globular head with three Fabs recognizing an F trimer. The six Fab CDRs and FRL3 contribute to burying roughly $1,000 \AA^{2}$ at the interface with F with equal contributions of the 1F5 heavy and light chains (Fig. 3d). The 1F5 paratope is dominated by aromatic amino acid residues, which contact $\mathrm{F}$ through hydrogen bonding and shape complementarity, including heavy chain

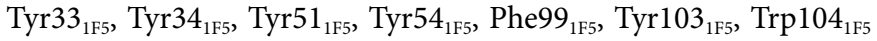
and light chain Tyr31 $1_{1 F 5}$, Tyr $48_{1 F 5}$, Trp $90_{1 F 5}$, Tyr91 $1 F 5$, Tyr93 $3_{1 F 5}$ (Fig. 3e). CDRL2 and CDRH3 interact electrostatically with the HeV F HRA $\beta$-hairpin through contacts involving the Glu166 ${ }_{\mathrm{HeV}}$ side chain carboxylate and the CDRL2 Ser55 $5_{1 F 5}$ and CDRH3 Tyr108 $8_{1 F 5}$ side chain hydroxyl groups as well as through the Lys $160_{\mathrm{HeV}}$ amine and CDRL2 Asn52 $2_{1 F 5}$ amide side chains. CDRH1-H3 and CDRL1-L3 recognize residues of both the $F_{1}$ and $F_{2}$ subunits in the core $\beta$-sheet and the region $\mathrm{C}$ terminal of the central helix through electrostatic contacts such as (1) the CDRH2 Thr53 $3_{1 \mathrm{~F} 5}$ hydroxyl and Asn51 $1_{\mathrm{HeV}}$ amide side chains, (2) the CDRH3 Ser $101_{1 \mathrm{~F} 5}$ hydroxyl and the Thr5 $4_{\mathrm{HeV}}$ backbone carbonyl, (3) the CDRL1 Tyr31 $1_{1 \mathrm{~F} 5}$ hydroxyl and the Arg244 $4_{\mathrm{HeV}}$ backbone carbonyl and (4) the CDRL3 Tyr91 ${ }_{1 \mathrm{~F} 5}$ backbone carbonyl and Tyr93 ${ }_{1 \mathrm{~F} 5}$ backbone amide with the Glu251 $1_{\mathrm{HeV}}$ backbone amide and side chain carboxylate, respectively. Finally, CDRL1 and FRL3 bridge the interface between two protomers by contacting the upstream helix of a neighboring F protomer (Fig. 3e).

Analysis of the 1F5 epitope conservation between NiV F and $\mathrm{HeV} \mathrm{F}$ shows that virtually all residues buried by $1 \mathrm{~F} 5$ binding are strictly conserved (Fig. 3f). Variable positions are $\mathrm{Thr} 74_{\mathrm{HeV}}$ and Ser $81_{\mathrm{HeV}}$, which are conservatively substituted to $S e r 74_{\mathrm{NiV}}$ and Thr81 $1_{\mathrm{Niv}}$, respectively. These two amino acid residue differences are not expected to have a major impact on the epitope-paratope interface, in support of the observations that the 1F5 Fab recognizes both $\mathrm{NiV} \mathrm{F}$ and $\mathrm{HeV} F$ trimers with near-identical affinities (Figs. $3 \mathrm{f}$ and 1c, d and Supplementary Table 1).

Comparisons of the structures of $\mathrm{HeV} \mathrm{F}$ in complex with 1F5 and of NiV F bound to $5 \mathrm{~B} 3$ shows that the two neutralizing Abs recognize a very similar region on the $\mathrm{F}$ trimer through equal contributions of the heavy and light chains to their paratopes. The two Fabs, however, are rotated roughly $130^{\circ}$ relative to each other, which results in almost complete inversion of the roles of the heavy and light chains for F recognition (Fig. 3g). Due to the different angles of approach between the two Fabs, the HeV/1F5 HRA $\beta$-hairpin adopts a conformation more similar to the apo $\mathrm{HeV} \mathrm{F}^{16}$ and $\mathrm{NiV} \mathrm{F}^{17}$ structures than to the NiV F-5B3 structure ${ }^{31}$.

Structure-guided design of F-targeting Ab cocktails. As virus passaging in the presence of subneutralizing concentrations of Abs can lead to the emergence of neutralization-escape mutants ${ }^{31}$, we tested this possibility using the 1F5 and the 12B2 Abs. Although we could not isolate escape mutants for 1F5 (in two independent laboratories), 12B2 selected for the S191R escape mutant that lies within the $12 \mathrm{~B} 2$ epitope. We therefore set out to formulate $\mathrm{Ab}$ cocktails

Fig. 3 | The 1F5 Ab recognizes a conserved quaternary epitope on the $\mathrm{HeV}$ glycoprotein. $\mathbf{a}, \mathbf{b}$, Ribbon diagrams showing two orthogonal views (a, side view; $\mathbf{b}$, top view) of the cryo-EM structure of the HeV F ectodomain trimer in complex with the 1F5 Fab fragment. One F protomer is rendered in teal and the other two protomers in gray. c, Molecular surface representation of the HeV F trimer with the CDR loops of a single $1 F 5$ Fab shown as ribbons, highlighting the quaternary nature of the epitope. $\mathbf{d}$, Contribution of distinct regions of $1 F 5$ to epitope recognition at the surface of HeV F. CDRH, heavy chain CDR; CDRL, light chain CDR; HFR, heavy chain framework region; LFR, light chain framework region. e, Zoomed-in view of the interface between $\mathrm{HeV} \mathrm{F}$ and $1 F 5$ with selected residues rendered as sticks. One protomer of HeV F is colored in teal and an adjacent protomer in gray. In a-d, the 1F5 variable heavy $\left(\mathrm{VH}_{1 \mathrm{~F} 5}\right)$ and light $\left(\mathrm{VL}_{1 \mathrm{F5}}\right)$ chains are colored purple and pink, respectively. $\mathbf{f}$, Molecular surface representation of the HeV $\mathrm{F}$ trimer showing the 1F5 footprint colored by residue conservation between HeV F and NiV F glycoproteins. Conservative sub, conservative substitution. $\mathbf{g}$, Superimposition of the 1F5-bound and 5B3-bound HeV F and NiV F structures, respectively, showing that the two Fabs adopt distinct orientations to recognize largely overlapping epitopes. The variable light chain (VL) and variable heavy chain (VH) of 5B3 are colored yellow and gold, respectively. The VL and VH of $1 F 5$ are colored pink and purple, respectively. 
a

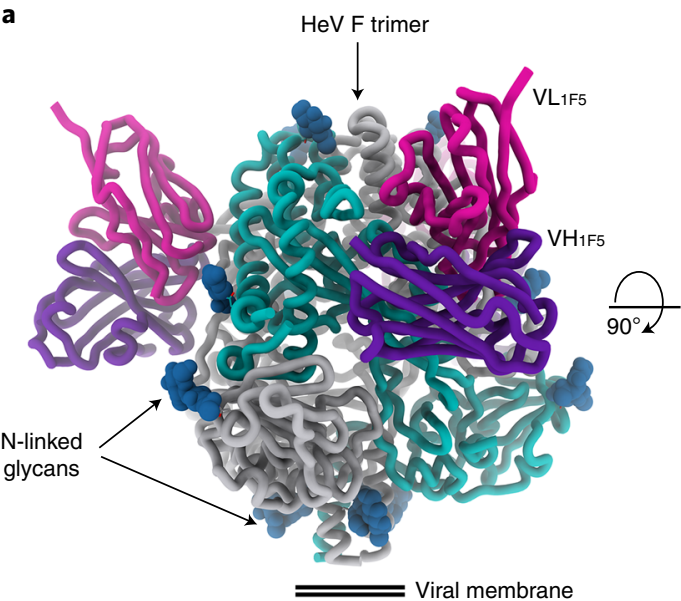

c

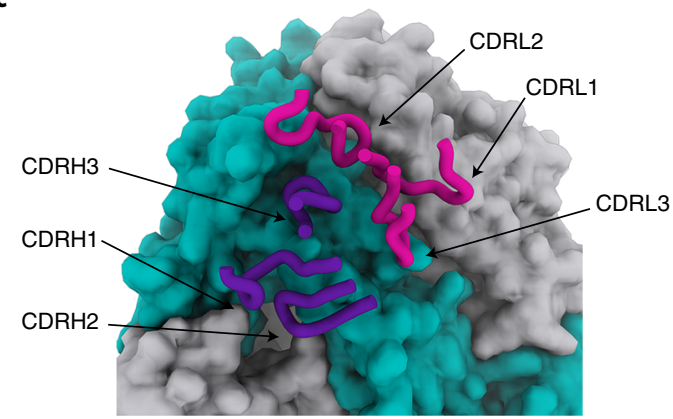

d

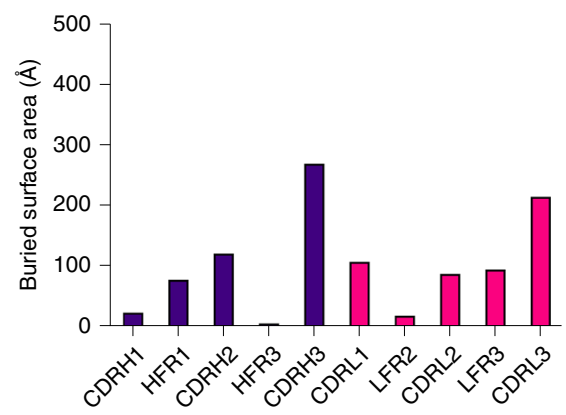

f

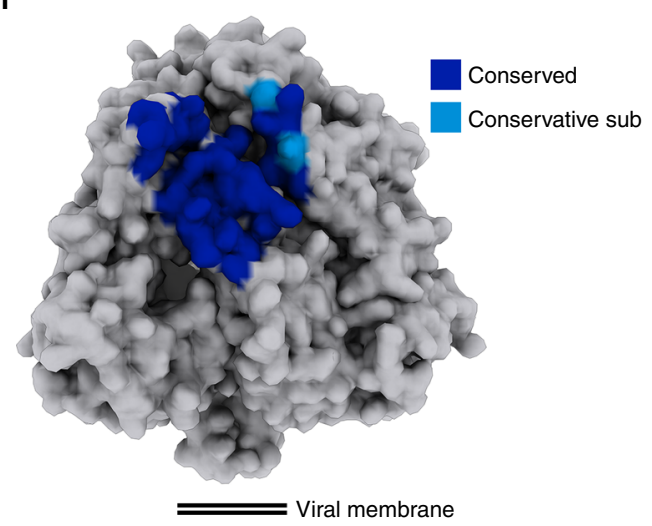

b

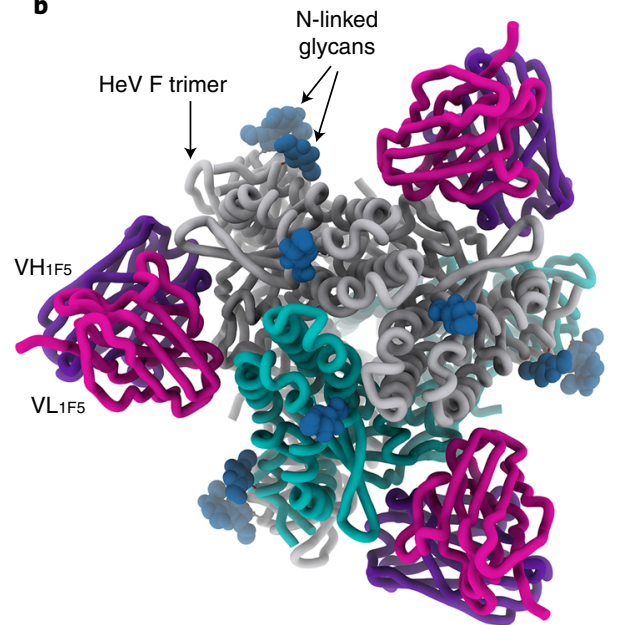

e

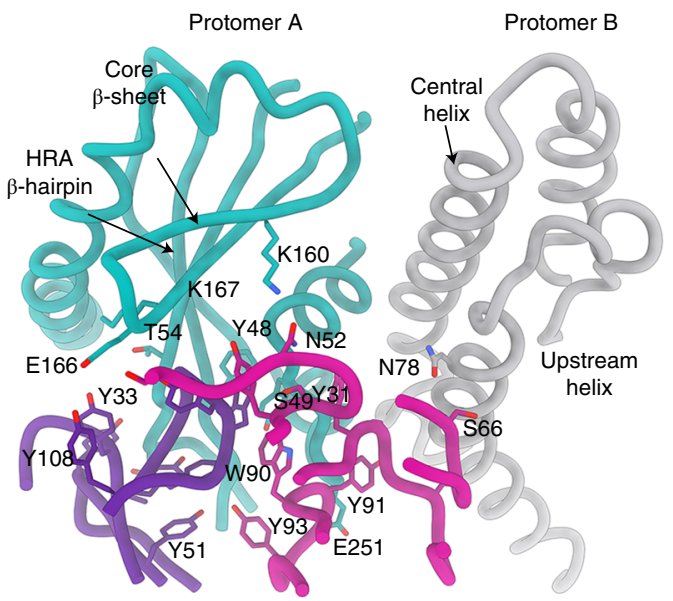

g

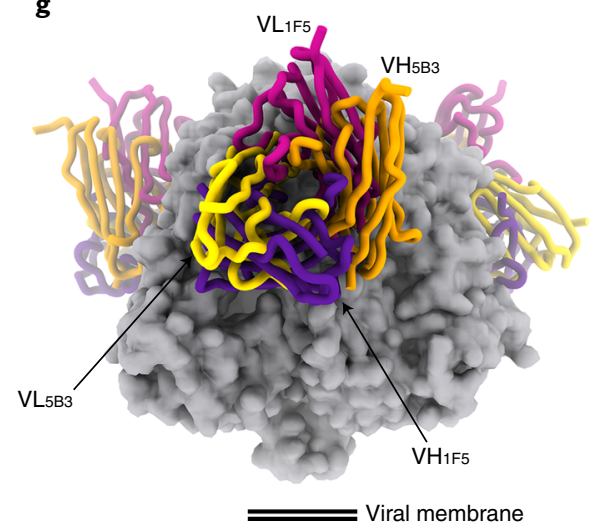

combining the benefits of multiple monoclonal Abs based on the observation that 12B2 engages the F apex whereas 1F5 (and the previously characterized $5 \mathrm{~B} 3$, ref. ${ }^{31}$ ) binds almost perpendicularly to the $\mathrm{F}$ threefold molecular axis and radiates from its side. Superimposition of the NiV F-12B2 and NiV F-5B3 structures indicates that no steric hindrance would occur between the variable domains (or the Fab fragments) as a result of binding to the same F protomer within a trimer. Superimposition of the HeV F-1F5 and NiV F-12B2 structures, however, suggests the framework of the two-Fab variable domains could sterically clash on recognition of a 
single $\mathrm{F}$ protomer within the trimer although they bind to entirely distinct epitopes (Fig. 4a,b).

We therefore assessed whether combinations of two Fabs targeting different $\mathrm{F}$ regions could simultaneously interact with $\mathrm{NiV} F$ by determining three-dimensional (3D) EM reconstructions of the following two negative-stained ternary complexes: NiV F-12B2-5B3 and NiV F-12B2-1F5. The resulting EM structures suggest F can simultaneously interact with the $12 \mathrm{~B} 2$ and $5 \mathrm{~B} 3 \mathrm{Fabs}$ or with the $12 \mathrm{~B} 2$ and 1F5 Fabs, indicating these Ab cocktails should be further evaluated (Supplementary Fig. 4). The ability of F to simultaneously engage $12 \mathrm{~B} 2$ and $1 \mathrm{~F} 5$ or $12 \mathrm{~B} 2$ and $5 \mathrm{~B} 3$ was further corroborated in a competitive binding experiment using BLI (Fig. 4c). After saturation with either 1F5 or 5B3 Fab during the association phase $1, \mathrm{NiV}$ F could subsequently recognize the 12B2 Fab fragment as observed during the association phase 2 . The larger magnitude of the binding signal observed for 12B2 binding to the F-5B3 complex compared to the F-1F5 complex (Fig. 4c) supports our structural data and likely resulted from steric hindrance between the 12B2 and 1F5 Fabs on recognition of the same $\mathrm{F}$ protomer within the trimer.

Using the aforementioned split-luciferase-reporter-based cellcell fusion assay, we observed that Ab cocktails comprising 12B2 and either $5 \mathrm{~B} 3$ or $1 \mathrm{~F} 5$ have the same inhibitory effect as single $\mathrm{mAb}$ treatment on HNV F/G-mediated membrane fusion (Figs. 4d,e and $1 \mathrm{e}, \mathrm{f})$. Next, we compared the neutralization potency and breadth of the humanized mAb cocktails, h12B2-h5B3.1 and h12B2-h1F5, to the three individual Abs by plaque reduction assays. The different data points were obtained by using either a given concentration of a single $\mathrm{Ab}$ or half the concentration of the two Abs combined to allow side-by-side comparison. Collectively, the data show that both Ab cocktails can neutralize $\mathrm{NiV}-\mathrm{M}, \mathrm{NiV}-\mathrm{B}$ and $\mathrm{HeV}$ in vitro (Fig. $4 \mathrm{f}$ and Supplementary Table 2). Kruskal-Wallis tests show that there is no statical difference between the potency of each mAb cocktails compared with those of each component mAb (Supplementary Table 2 and Methods). These findings provide proof-of-concept that neutralizing Abs recognizing distinct $F$ epitopes could be used in combination to inhibit $\mathrm{NiV}$ and $\mathrm{HeV}$ entry into host cells and are likely to prevent or limit the emergence of neutralization-escape mutants.

\section{Discussion}

Ab cocktails represent a promising strategy to combat emerging and reemerging RNA viruses that can exhibit high mutation rates contributing to immune evasion. Administering multiple Abs simultaneously can mimic the benefits of natural polyclonal $\mathrm{Ab}$ responses, such as neutralization breadth and potency as well as reduction of the frequency of emergence of escape mutants, in a fully tailored and controllable manner. REGN-EB3 is an example of an Ab cocktail

Fig. 4 | Formulation of F-targeted Abs cocktails against $\mathrm{NiV}$ and $\mathrm{HeV}$. a, Superimposition of the NiV F-12B2 and HeV F-1F5 structures shows a potential clash between the framework regions of the two Fabs. b, Superimposition of the NiV F-12B2 and NiV F-5B3 structures (PDB 6TYS) indicates that binding of the two Fabs is compatible without clash.

c, Analysis of competitive binding of 12B2 Fab to preformed NiV F-1F5 or NiV F-5B3 complexes by BLI. Each amine-coupled NiV F biosensor probe was sequentially dipped in solution containing $600 \mathrm{nM} 1 \mathrm{~F} 5$ or $5 \mathrm{~B} 3 \mathrm{Fab}$ and then 1,200 nM 12B2 Fab +600 nM 1F5 (purple) or 1,200 nM 12B2 Fab +600 nM $5 B 3$ Fab (pink). d,e, 12B2-1F5 and 12B2-5B3 Ab cocktails inhibit membrane fusion mediated by NiV (d) and HeV (e) glycoproteins. D40 is an Ab against HIV gp-41 used as a negative control. $\mathbf{f}$, Neutralization of authentic NiV-M, NiV-B and HeV by h1F5, h5B3.1, h12B2, h1F5-h12B2 and h5B3.1h12B2. Data for h12B2, h1F5, h12B2-h1F5 and h12B2-h5B3.1 shown are from one replicate of two independent experiments with independent virus preparations. Data for h5B3.1 are reproduced from Dang et al. ${ }^{31} .{ }^{\prime} C_{50}$ values for each replicate experiment are reported in Supplementary Table 2. providing complete in vivo protection of nonhuman primates from a lethal dose of Ebola virus ${ }^{43}$ and has been shown to provide substantial protection in a phase 3 study in patients infected with Ebola virus $^{44}$ and was recently approved by the FDA. MBP $134^{\mathrm{AF}}$ is a more recently developed $\mathrm{Ab}$ cocktail that provides protection of nonhuman primates against Ebola virus, Sudan virus and Bundibugyo virus after injection of a single dose up to several days postinfection $^{45,46}$. Furthermore, several studies have suggested the use of well-characterized $\mathrm{Ab}$ cocktails as a replacement for polyclonal

a

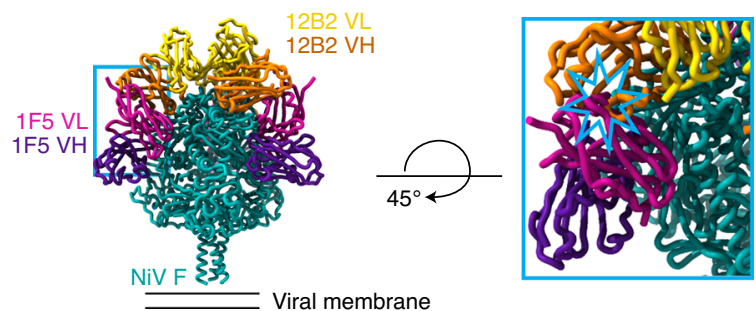

b
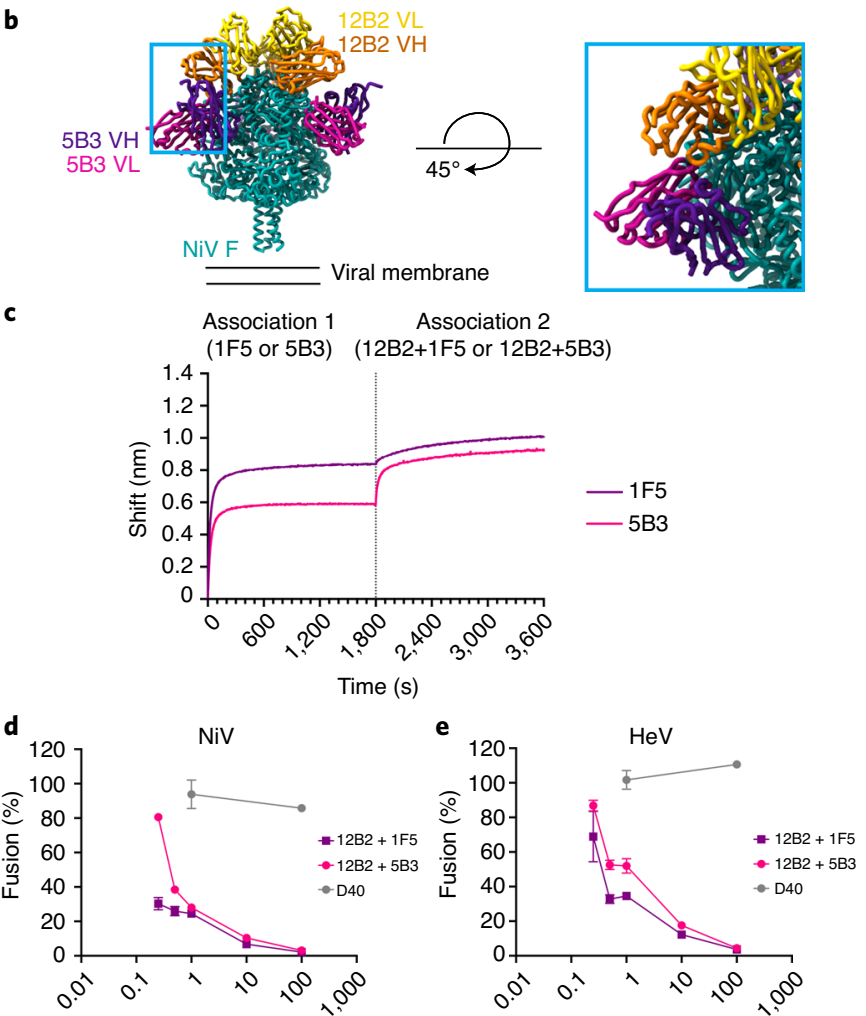

MAb concentration $\left(\mu \mathrm{g} \mathrm{ml}^{-1}\right)$

MAb concentration $\left(\mu \mathrm{g} \mathrm{ml}^{-1}\right.$ )

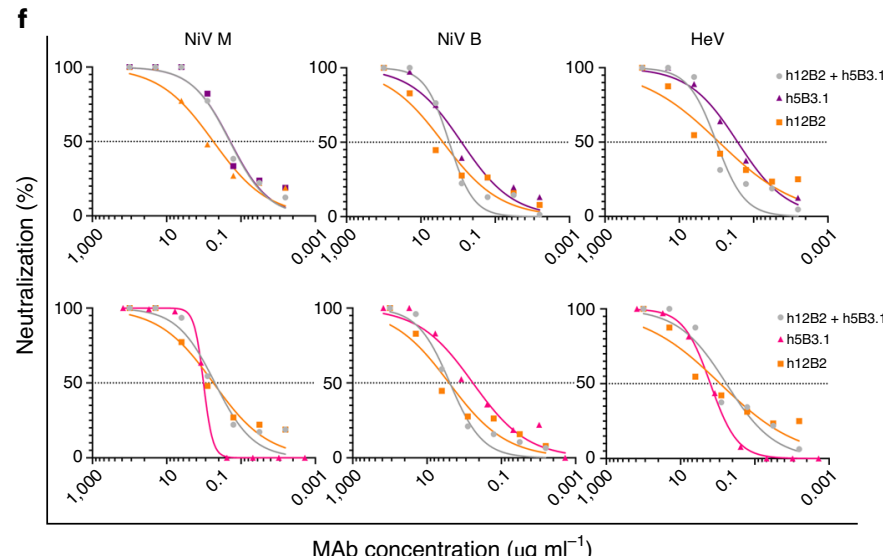


human or equine Abs, which are currently used for postexposure treatment of rabies ${ }^{47-49}$.

The current lack of approved HNV vaccines or drugs for humans along with the compassionate use of the m102.4 Ab in individuals with high-risk exposure to $\mathrm{NiV}$ and $\mathrm{HeV}$ emphasizes the urgent need for prophylactic and/or therapeutic solutions ${ }^{50}$. m102.4 potently neutralizes $\mathrm{NiV}$ and $\mathrm{HeV}$ through competitive inhibition of G-mediated viral attachment to the host receptors, ephrin-B2 or ephrin-B3 (refs. ${ }^{3-7}$ ). In addition to m102.4, there has only been two other neutralizing anti-HNVs mAbs isolated and characterized in depth: h5B3.1 (ref. ${ }^{31}$ ) and mAb66 (ref. ${ }^{51}$ ). Using high-resolution cryo-EM, we characterized two neutralizing Abs recognizing distinct, yet highly conserved, epitopes on the surface of the NiV F and $\mathrm{HeV} \mathrm{F}$ glycoproteins, adding to the collection of Abs to be used against these dangerous viruses. $1 \mathrm{~F} 5$ (such as $5 \mathrm{~B} 3$, ref. ${ }^{31}$ ) engages the side of the $\mathrm{F}$ trimer, whereas $12 \mathrm{~B} 2$ binds to a new epitope near the $\mathrm{F}$ apex distal to the viral membrane. All three Abs recognize quaternary epitopes (involving interactions with the HRA region) and possibly staple $\mathrm{F}$ in the prefusion state (Supplementary Fig. 5ad) to block viral entry by preventing membrane fusion. However, $1 \mathrm{~F} 5$ and $12 \mathrm{~B} 2$ are unlikely to cross-react with $\mathrm{F}$ proteins from Henipa-related viruses such as CedV, GhV and Mojiang virus due to the low level of similarity in the amino acids constituting the epitopes of these two mAbs on these F proteins compared to $\mathrm{NiV}$ and $\mathrm{HeV} F$ (Supplementary Fig. 5e).

In this study, we also successfully generated humanized versions of $12 \mathrm{~B} 2$ and 1F5 with equivalent neutralization potency to the parental murine versions. Our structural data demonstrate that $12 \mathrm{~B} 2$ and $5 \mathrm{~B} 3$ or $12 \mathrm{~B} 2$ and $1 \mathrm{~F} 5$ can simultaneously interact with $\mathrm{F}$, which led us to investigate the use of Ab cocktails on HNV neutralization. Our data show that both h12B2-5B3.1 and h12B2-h1F5 humanized $\mathrm{mAb}$ cocktails have a neutralization potency comparable to each individual $\mathrm{Ab}$ component. These two-Ab cocktails could be tailored to include 3 mAbs: m102.4, to prevent receptor engagement at the host cell surface, and $12 \mathrm{~B} 2$ with $1 \mathrm{~F} 5$ or $5 \mathrm{~B} 3$, to inhibit F-mediated membrane fusion. Such a trivalent cocktail would inhibit multiple viral functions involved in host cell entry, and limit further the risk of emergence of viral mutants escaping neutralization by targeting multiple different epitopes. We therefore expect that cocktail $\mathrm{Ab}$ therapies will emerge as promising effective countermeasure against HNVs and should be explored in future in vivo protection studies.

\section{Online content}

Any methods, additional references, Nature Research reporting summaries, source data, extended data, supplementary information, acknowledgements, peer review information; details of author contributions and competing interests; and statements of data and code availability are available at https://doi.org/10.1038/ s41594-021-00584-8.

Received: 22 October 2020; Accepted: 17 March 2021; Published online: 29 April 2021

\section{References}

1. Eaton, B. T., Broder, C. C., Middleton, D. \& Wang, L. F. Hendra and Nipah viruses: different and dangerous. Nat. Rev. Microbiol. 4, 23-35 (2006).

2. Luby, S. P. \& Gurley, E. S. Epidemiology of Henipavirus disease in humans. Curr. Top. Microbiol. Immunol. 359, 25-40 (2012).

3. Gurley, E. S. et al. Convergence of humans, bats, trees, and culture in Nipah virus transmission, Bangladesh. Emerg. Infect. Dis. 23, 1446-1453 (2017).

4. Halpin, K. et al. Pteropid bats are confirmed as the reservoir hosts of henipaviruses: a comprehensive experimental study of virus transmission. Am. J. Tropical Med. Hyg. 85, 946-951 (2011).

5. Clayton, B. A. Nipah virus: transmission of a zoonotic paramyxovirus. Curr. Opin. Virol. 22, 97-104 (2017).

6. Pernet, O. et al. Evidence for henipavirus spillover into human populations in Africa. Nat. Commun. 5, 5342 (2014)
7. Navaratnarajah, C. K., Generous, A. R., Yousaf, I. \& Cattaneo, R. Receptor-mediated cell entry of paramyxoviruses: mechanisms, and consequences for tropism and pathogenesis. J. Biol. Chem. 295, 2771-2786 (2020)

8. Bowden, T. A. et al. Structural basis of Nipah and Hendra virus attachment to their cell-surface receptor ephrin-B2. Nat. Struct. Mol. Biol. 15, 567-572 (2008).

9. $\mathrm{Xu}, \mathrm{K}$. et al. Host cell recognition by the henipaviruses: crystal structures of the Nipah G attachment glycoprotein and its complex with ephrin-B3. Proc. Natl Acad. Sci. USA 105, 9953-9958 (2008).

10. Negrete, O. A. et al. EphrinB2 is the entry receptor for Nipah virus, an emergent deadly paramyxovirus. Nature 436, 401-405 (2005).

11. Bonaparte, M. I. et al. Ephrin-B2 ligand is a functional receptor for Hendra virus and Nipah virus. Proc. Natl Acad. Sci. USA 102, 10652-10657 (2005).

12. Negrete, O. A. et al. Two key residues in EphrinB3 are critical for its use as an alternative receptor for Nipah virus. PLoS Pathog. 2, e7 (2006).

13. Bishop, K. A. et al. Identification of Hendra virus $G$ glycoprotein residues that are critical for receptor binding. J. Virol. 81, 5893-5901 (2007).

14. Pager, C. T. \& Dutch, R. E. Cathepsin L is involved in proteolytic processing of the Hendra virus fusion protein. J. Virol. 79, 12714-12720 (2005).

15. Pager, C. T., Craft, W. W., Patch, J. \& Dutch, R. E. A mature and fusogenic form of the Nipah virus fusion protein requires proteolytic processing by cathepsin L. Virology 346, 251-257 (2006).

16. Wong, J. J. W., Paterson, R. G., Lamb, R. A. \& Jardetzky, T. S. Structure and stabilization of the Hendra virus F glycoprotein in its prefusion form. Proc. Natl Acad. Sci. USA 113, 1056-1061 (2016).

17. Xu, K. et al. Crystal structure of the pre-fusion Nipah virus fusion glycoprotein reveals a novel hexamer-of-trimers assembly. PLoS Pathogens 11, e1005322 (2015).

18. Chan, Y.-P. et al. Biochemical, conformational, and immunogenic analysis of soluble trimeric forms of henipavirus fusion glycoproteins. J. Virol. 86, 11457-11471 (2012).

19. Liu, Q. et al. Nipah virus attachment glycoprotein stalk C-terminal region links receptor binding to fusion triggering. J. Virol. 89, 1838-1850 (2015).

20. Liu, Q. et al. Unraveling a three-step spatiotemporal mechanism of triggering of receptor-induced Nipah virus fusion and cell entry. PLoS Pathogens $\mathbf{9}$, e1003770 (2013)

21. Connolly, S. A., Leser, G. P., Yin, H. S., Jardetzky, T. S. \& Lamb, R. A. Refolding of a paramyxovirus $\mathrm{F}$ protein from prefusion to postfusion conformations observed by liposome binding and electron microscopy. Proc. Natl Acad. Sci. USA 103, 17903-17908 (2006).

22. Wong, J. J. W. et al. Monomeric ephrinB2 binding induces allosteric changes in Nipah virus $G$ that precede its full activation. Nat. Commun. 8, 781 (2017)

23. Yin, H. S., Paterson, R. G., Wen, X., Lamb, R. A. \& Jardetzky, T. S. Structure of the uncleaved ectodomain of the paramyxovirus (hPIV3) fusion protein. Proc. Natl Acad. Sci. USA 102, 9288-9293 (2005).

24. Bossart, K. N. et al. A Hendra virus G glycoprotein subunit vaccine protects African green monkeys from Nipah virus challenge. Sci. Transl. Med. 4, 146ra107 (2012).

25. Bossart, K. N. et al. A neutralizing human monoclonal antibody protects against lethal disease in a new ferret model of acute Nipah virus infection. PLoS Pathogens 5, e1000642 (2009).

26. Geisbert, T. W. et al. Therapeutic treatment of Nipah virus infection in nonhuman primates with a neutralizing human monoclonal antibody. Sci. Trans. Med. 6, 242ra82 (2014).

27. Zhu, Z. et al. Exceptionally potent cross-reactive neutralization of nipah and hendra viruses by a human monoclonal antibody. J. Infect. Dis. 197, 846-853 (2008)

28. Zhu, Z. et al. Potent neutralization of Hendra and Nipah viruses by human monoclonal antibodies. J. Virol. 80, 891-899 (2006).

29. $\mathrm{Xu}, \mathrm{K}$. et al. Crystal structure of the Hendra virus attachment $\mathrm{G}$ glycoprotein bound to a potent cross-reactive neutralizing human monoclonal antibody. PLoS Pathogens 9, e1003684 (2013).

30. Playford, E. G. et al. Safety, tolerability, pharmacokinetics, and immunogenicity of a human monoclonal antibody targeting the $\mathrm{G}$ glycoprotein of henipaviruses in healthy adults: a first-in-human, randomised, controlled, phase 1 study. Lancet Infect. Dis. 20, 445-454 (2020).

31. Dang, H. V. et al. An antibody against the F glycoprotein inhibits Nipah and Hendra virus infections. Nat. Struct. Mol. Biol. 26, 980-987 (2019).

32. Mire, C. E. et al. A cross-reactive humanized monoclonal antibody targeting fusion glycoprotein function protects ferrets against lethal Nipah virus and Hendra virus infection. J. Infect. Dis. 221, S471-S479 (2020).

33. Drexler, J. F. et al. Bats host major mammalian paramyxoviruses. Nat. Commun. 3, 796 (2012).

34. Wu, Z. et al. Novel Henipa-like virus, Mojiang paramyxovirus, in rats, China, 2012. Emerg. Infect. Dis. 20, 1064-1066 (2014).

35. Laing, E. D. et al. Structural and functional analyses reveal promiscuous and species specific use of ephrin receptors by Cedar virus. Proc. Natl Acad. Sci. USA 116, 20707-20715 (2019). 
36. Kondo, N., Miyauchi, K., Meng, F., Iwamoto, A. \& Matsuda, Z. Conformational changes of the HIV-1 envelope protein during membrane fusion are inhibited by the replacement of its membrane-spanning domain. J. Biol. Chem. 285, 14681-14688 (2010)

37. Tortorici, M. A. et al. Ultrapotent human antibodies protect against SARS-CoV-2 challenge via multiple mechanisms. Science https://doi org/10.1126/science.abe3354 (2020).

38. Pinto, D. et al. Cross-neutralization of SARS-CoV-2 by a human monoclonal SARS-CoV antibody. Nature 583, 290-295 (2020).

39. Walls, A. C. et al. Unexpected receptor functional mimicry elucidates activation of coronavirus fusion. Cell 176, 1026-1039.e15 (2019).

40. Borst, A. J. et al. Germline VRC01 antibody recognition of a modified clade C HIV-1 envelope trimer and a glycosylated HIV-1 gp120 core. Elife 7, e37688 (2018).

41. Stewart-Jones, G. B. E. et al. Trimeric HIV-1-Env structures define glycan shields from clades A, B, and G. Cell 165, 813-826 (2016).

42. West, B. R. et al. Structural basis of pan-Ebolavirus neutralization by a human antibody against a conserved, yet cryptic epitope. Mbio 9, e01674-18 (2018).

43. Pascal, K. E. et al. Development of clinical-stage human monoclonal antibodies that treat advanced ebola virus disease in nonhuman primates. J. Infect. Dis. 218, S612-S626 (2018).

44. Mulangu, S. et al. A randomized, controlled trial of ebola virus disease therapeutics. N. Engl. J. Med. 381, 2293-2303 (2019).

45. Bornholdt, Z. A. et al. A two-antibody pan-Ebolavirus cocktail confers broad therapeutic protection in ferrets and nonhuman primates. Cell Host Microbe 25, 49-58.e5 (2019).
46. Wec, A. Z. et al. Development of a human antibody cocktail that deploys multiple functions to confer pan-ebolavirus protection. Cell Host Microbe 25, 39-48.e5 (2019).

47. Logtenberg, T. Antibody cocktails: next-generation biopharmaceuticals with improved potency. Trends Biotechnol. 25, 390-394 (2007).

48. Chao, T.-Y. et al. SYN023, a novel humanized monoclonal antibody cocktail, for post-exposure prophylaxis of rabies. PLoS Negl. Trop. Dis. 11, e0006133 (2017)

49. Nagarajan, T. et al. in Human Antibody Therapeutics for Viral Disease (ed. Dessain, S. K.) 67-101 (Springer, 2008); https://doi. org/10.1007/978-3-540-72146-8_3

50. Amaya, M. \& Broder, C. C. Vaccines to emerging viruses: Nipah and Hendra. Annu. Rev. Virol. 7, 447-473 (2020).

51. Avanzato, V. A. et al. A structural basis for antibody-mediated neutralization of Nipah virus reveals a site of vulnerability at the fusion glycoprotein apex. Proc. Natl Acad. Sci. USA 116, 25057-25067 (2019).

52. Earl, P. L., Broder, C. C., Doms, R. W. \& Moss, B. Epitope map of human immunodeficiency virus type 1 gp41 derived from 47 monoclonal antibodies produced by immunization with oligomeric envelope protein. J. Virol. 71, 2674-2684 (1997).

Publisher's note Springer Nature remains neutral with regard to jurisdictional claims in published maps and institutional affiliations.

(C) The Author(s), under exclusive licence to Springer Nature America, Inc. 2021 


\section{Methods}

Cell lines. Human embryonic kidney $293 \mathrm{~F}$ cells (Life Technologies) were grown in 293FreeStyle expression medium (Life Technologies), cultured at $37^{\circ} \mathrm{C}$ with $8 \%$ $\mathrm{CO}_{2}$ and 150 r.p.m.

PgsA-745 (ATCC CRL-2242) cells, a derivative deficient in xylosyltransferase (UDP-D-xylose/serine-1,3-D-xylosyltransferase), referred to as CHO745 in this study, was maintained at $37^{\circ} \mathrm{C}$ with $5 \% \mathrm{CO}_{2}$ in Gibco F-12 Nutrient $\mathrm{K}$ (Kaighn's Modification of Ham's F-12) medium (ThermoFisher Scientific) supplemented with $10 \%$ fetal bovine serum (FBS) (ThermoFisher Scientific).

$\mathrm{CHO}$ cells do not express B-class ephrins endogenously ${ }^{53}$ and $\mathrm{CHO} 745$ cells were used to generate a stable cell line expressing ephrin-B2 (CHO745-ephrin-B2) by transfection of an expression plasmid encoding an S-tagged ephrin-B2 $\mathrm{ORF}^{35}$ and selected by hygromycin B resistance. CHO745-ephrin-B2 cells were maintained in the same media as $\mathrm{CHO} 745$ supplemented with $800 \mu \mathrm{g} \mathrm{ml}^{-1}$ hygromycin B.

NiV F and HeV F soluble ectodomain construct. The NiV F and $\mathrm{HeV} \mathrm{F}$ ectodomain constructs include the codon optimized NiV F (isolate UMMC1, GenBank Sequence accession no. AY029767) or HeV F (isolate Horse/Australia/ Hendra/1994) ectodomain (residues 1-487) fused to a C-terminal GCNt followed by a factor Xa sequence and an S-tag (KLKETAAAKFERQHMDS) cloned in a pcDNA Hygro (+)-CMV + vector for transient expression in FreeStyle 293F cells. $\mathrm{NiV}$ F S69A ectodomain mutant was generated via site-directed mutagenesis. All mutation-containing constructs were sequence verified.

mAb sequencing by mass spectrometry. The primary structures of the $12 \mathrm{~B} 2$ and 1F5 heavy and light chains were determined by liquid chromatography-mass spectrometry (LC-MS, Rapid Novor, Inc.). For each Ab, eight in-solution and in-gel digestions were prepared using five different enzymes (pepsin, trypsin, chymotrypsin, Asp N, Lys C). The in-solution digestions for each sample were processed with disulfide reduction, alkylation and then enzyme digestion. Each digestion contained peptides from both heavy and light immunoglobulin chains. The in-gel digestions were prepared for immunoglobulin chains after gel separation. The sample was processed with disulfide reduction, gel separation, deglycosylation, disulfide reduction a second time, alkylation and then digestion. Digestions were analyzed by LC-tandem MS using ThermoFisher Q ExactiveTM, Orbitrap FusionTM mass spectrometers. Peptides were characterized from LC-tandem MS data using de novo peptide sequencing and then assembled into antibody sequences.

mAb Humanization. Humanization of mAbs 1F5 and 12B2 was performed using Mapp Biopharmaceutical's in silico humanization pipeline. For each mAb, mouse constant heavy and light chain domains were exchanged with human sequences. High-resolution homology models were constructed of each variable domain, and a structure-guided resurfacing approach was employed to maintain the murine CDR conformations and antigen binding while reducing deviations from the inferred human germline progenitor sequences. Initial humanization was followed by iterative rounds of structure-guided engineering to eliminate residues that contribute to chemical liability hotspots such as aggregation, glycosylation and enzymatic cleavage.

Generation of 12B2 and 1F5 humanized IgGs. Humanized 12B2 and 1F5 IgGs were produced by transient expression in ExpiCHO cells (ThermoFisher Scientific) and purified via protein A chromatography. For each humanized mAb, cells were pelleted $8 \mathrm{~d}$ posttransfection by centrifugation for $30 \mathrm{~min}$ at $3,000 \mathrm{~g}$ and supernatant was collected for purification of soluble IgG. Supernatant was flowed over a $5 \mathrm{ml}$ protein A agarose column using an Akta Pure (MabSelect SuRe, GE Healthcare Life Sciences). The column was washed with five column volumes of Dulbecco's phosphate-buffered saline (Gibco) and then IgG was eluted with IgG Elution Buffer (Pierce). The elution fraction was then neutralized with Tris $\mathrm{pH} 9.0$ to a final $\mathrm{pH}$ of 7.5. The neutralized elution fraction for each $\mathrm{mAb}$ was then concentrated to $5 \mathrm{mg} \mathrm{ml}^{-1}$ using a $30 \mathrm{kDa}$ moleculer weight cutoff Amicon Ultra spin concentrator (Millipore), then aliquoted and stored at $-80^{\circ} \mathrm{C}$.

Generation of Fab fragments from IgG. The murine 1F5, 12B2 and 5B3 Fab fragments were obtained by fragmentation of the corresponding mouse IgGs using Pierce Mouse IgG1 Fab and F(ab')2 preparation kits according to the manufacturer's protocol. The Fab-containing fraction was further purified using a Superdex 75 10/300 gel filtration column equilibrated in a buffer containing $50 \mathrm{mM}$ Tris $\mathrm{pH} 8.0$ and $150 \mathrm{mM} \mathrm{NaCl}$ and stored at $4^{\circ} \mathrm{C}$.

$\mathrm{NiV} F$ and $\mathrm{HeV} F$ ectodomain production. Soluble $\mathrm{NiV} F$ and $\mathrm{HeV} F$ and the corresponding S69A mutants were produced by transient transfection of FreeStyle $293 \mathrm{~F}$ cells at a density of $1 \times 10^{6}$ cells per $\mathrm{ml}$ with the corresponding plasmid using 293-Free transfection reagent (Millipore) and Opti-MEM (ThermoFisher) according to the manufacturer's protocol. After $5 \mathrm{~d}$ in a humidified shaking incubator and maintained at $37^{\circ} \mathrm{C}$ and $8 \% \mathrm{CO}_{2}$, the cell supernatant was collected and clarified of cell debris by centrifugation. Subsequent affinity purification was carried out using S-protein agarose (Millipore Sigma, Novagen) and elution with $0.2 \mathrm{M}$ citric acid $\mathrm{pH} 2.0$ followed by immediate neutralization with $1.0 \mathrm{M}$ phosphate buffer $\mathrm{pH}$ 8.0. The eluted fraction was buffer-exchanged into $50 \mathrm{mM}$ phosphate-buffered saline (PBS) pH 7.4, $150 \mathrm{mM} \mathrm{NaCl}$ using a $30 \mathrm{kDa}$ cutoff centrifugal concentrator (Millipore).

BLI. Assays were performed with an Octet Red 96 instrument (ForteBio, Inc.) at $30^{\circ} \mathrm{C}$ while shaking at 1,000 r.p.m. All measurements were corrected by subtracting the background signal obtained from biosensors without immobilized $\mathrm{HeV} F$ or NiV F. S-peptide tagged HeV F WT, NiV F WT and NiV F S69A in PBS at pH 7.4 were diluted to $20 \mathrm{\mu g} \mathrm{ml}^{-1}$ in $10 \mathrm{mM}$ acetate buffer $\mathrm{pH} 5.0$ before immobilization on (NHS-EDC activated) Amine Reactive 2nd Generation (AR2G, ForteBio) biosensors for $300 \mathrm{~s}$. Sensors were then quenched in $1 \mathrm{M}$ ethanolamine (ForteBio) for $300 \mathrm{~s}$ and incubated in kinetics buffer ( $1 \times$ PBS, $0.001 \%$ BSA, $0.02 \%$ Tween 20 and $0.005 \% \mathrm{NaN}_{3}$ (ForteBio)) for $300 \mathrm{~s}$ to establish the baseline signal ( $\mathrm{nm}$ shift).

For kinetics assays, HeV F- or NiV F- or NiV F S69A-loaded AR2G sensors were then immersed into solutions of purified Fab (12B2 or 1F5) diluted in kinetics buffer to desired concentrations. Curve fitting was performed using a 1:1 binding model to determine binding kinetics with the ForteBio data analysis software. Mean $k_{\text {on }}$ and $k_{\text {off }}$ values were determined with a global fit applied to all data. The experiments were performed twice with independent NiV F, NiV F S69A and HeV F protein preparations yielding identical results and kinetic parameters. The estimated kinetics parameters for each replicate are summarized in Supplementary Table 1.

For sequential $\mathrm{mAb}$ binding assays, NiV F-loaded AR2G sensors were immersed into solutions of purified 1F5 or 5B3 Fab at $600 \mathrm{mM}$ in association phase 1 and then into two-Fab solutions containing $600 \mathrm{mM} 1 \mathrm{~F} 5+1,200 \mathrm{nM} 12 \mathrm{~B} 2$ Fabs or $600 \mathrm{mM} 5 \mathrm{~B} 3+1,200 \mathrm{nM} 12 \mathrm{~B} 2$ in association phase 2. All Fab solutions were diluted to the desired concentrations in kinetics buffer.

Cryo-EM specimen preparation and data collection. A sheet of $50 \times 75 \mathrm{~mm}$, 0.15-0.2-mm thick Muscovite mica (Electron Microscopy Sciences) was slit into half to obtain two sheets with half the thickness. Continuous home-made carbon sheets were made by depositing carbon from a sharpened graphite rod Grade 1 (Ted Pella) onto this mica sheet using a Cressington high vacuum Carbon208 coater system. The thickness of the carbon sheet was examined by eyes. The amorphous carbon sheet was floated by slowly lowering the carbon-coated mica sheet into a glass bowl filled with deionized water. A Cu 200 mesh C-flat grid, CF-1.2/1.3 or CF-2/2 (Protochips Inc.), was then dipped under the floated carbon sheet with a tweezer, picked up through the carbon sheet and gently placed on a piece of Whatman paper on a petri dish to dry overnight before glow discharging and sample application or storage in a dry cool place for further use.

$\mathrm{S}$-peptide-tagged NiV F was incubated with 12B2 Fab at 1:4 molar excess Fab and S-peptide-tagged $\mathrm{HeV}$ F with 1F5 Fab at 1:2 molar excess Fab. Both complexes were incubated overnight at $4^{\circ} \mathrm{C}$. Then, $3 \mu \mathrm{l}$ of each complex, S-tagged NiV F-12B2 Fab or S-tagged HeV F-1F5, at a concentration of $0.1 \mathrm{mg} \mathrm{ml}^{-1} \mathrm{~F}$ was applied onto glow-discharged C-flat, Cu 200 mesh, CF-1.2/1.3 or CF-2/2, respectively, holey carbon grids (Protochips Inc.) covered with a thin layer of continuous home-made carbon and incubated for $20 \mathrm{~s}$ on grids. Grids were then plunge-frozen in liquid ethane, cooled with liquid nitrogen, using an FEI MK4 Vitrobot with a $2.5 \mathrm{~s}$ blot time. The chamber was kept at $20^{\circ} \mathrm{C}$ and $100 \%$ humidity during the blotting process.

Data acquisition was carried out with the Leginon data collection software $\mathrm{e}^{54}$ on an FEI Titan Krios electron microscope operated at $300 \mathrm{kV}$ and equipped with a Gatan BioQuantum energy filter (slit width of $20 \mathrm{eV}$ ) and a Gatan K2 Summit camera. The nominal magnification was $\times 130,000$ and the pixel size was $1.05 \AA$. The dose rate was adjusted to eight counts per pixel per $s$ and each video was acquired in super-resolution mode fractionated in 50 frames of $200 \mathrm{~ms}$ each. A summary of data collection is provided in Supplementary Table 2.

Cryo-EM data processing. For both NiV F-12B2 and HeV F-1F5 datasets, video frame alignment was carried out with motioncor2 (ref. ${ }^{55}$ ). Particles were automatically selected using DoGPicker within the Appion interface ${ }^{56}$. Initial defocus parameters were estimated using $\mathrm{GCTF}^{57}$. The picked particles were extracted and processed with a box size of 352 pixels $^{2}$ and preprocessed using Relion 3.0 (ref. ${ }^{58}$ ). Reference-free two-dimensional (2D) classification with Relion or CryoSPARC was used to select a subset of particles, which were used to generate an initial model using the ab initio reconstruction with no symmetry function in CryoSPARC ${ }^{59}$. This $3 \mathrm{D}$ map was subsequently used as a reference for running $3 \mathrm{D}$ classification within Relion on the entire dataset. A subset of particles was selected from these 3D classes for 3D refinement using Relion. Contrast transfer function (CTF) refinement in Relion 3.0 was performed to refine per-particle defocus values and particle images were subjected to the Bayesian polishing procedure in Relion 3.0 (ref. ${ }^{60}$ ) and 3D refinement before performing another round of CTF refinement and $3 \mathrm{D}$ refinement. The particles were subsequently subjected to another round of 3D classification in Relion 3.0 without refining angles and shifts. Particles from the best class were used for nonuniform refinement in CryoSPARC ${ }^{61}$ to obtain the final $3 \mathrm{D}$ reconstruction. Reported resolutions are based on the gold-standard Fourier shell correlation (FSC) of 0.143 criterion $^{62,63}$ and FSC curves were corrected for the effects of soft masking by high-resolution noise substitution ${ }^{64}$. Local resolution 
estimation and filtering was carried out using CryoSPARC. See Supplementary Figs. $1 \mathrm{e}$ and $3 \mathrm{e}$ for a detailed flow chart of data processing for each dataset.

Model building and analysis. UCSF Chimera ${ }^{65}$ was used to rigid-body fit the cryo-EM structures of the NiV F ectodomain (from PDB 6TYS) into NiV F-12B2 cryo-EM density and the prefusion $\mathrm{HeV} F$ crystal structure (PDB 5EJB) ${ }^{16}$ into the HeV F-1F5 cryo-EM density. The crystal structure of 5B3 Fab (PDB 6U1T) was rigid-body fit into the density for Fabs in the cryo-EM maps of NiV F-12B2 or $\mathrm{HeV} \mathrm{F-1F5.} \mathrm{The} \mathrm{1F5} \mathrm{and} \mathrm{12B2} \mathrm{Fab} \mathrm{fragments} \mathrm{were} \mathrm{then} \mathrm{rebuilt} \mathrm{manually} \mathrm{with} \mathrm{the}$ amino acid sequences obtained from mass spectrometry described above. Each model, NiV F-12B2 and HeV F-1F5, was subsequently rebuilt manually using $\operatorname{Coot}^{66}$ and refined using Rosetta ${ }^{67-69}$. Glycan refinement relied on a dedicated Rosetta protocol, which uses physically realistic geometries based on previous knowledge of saccharide chemical properties ${ }^{70}$ and was aided by using both sharpened and unsharpened maps. Models were analyzed using MolProbity ${ }^{71}$ EMRinger $^{72}$ and Privateer ${ }^{73}$. Figures were generated using UCSF ChimeraX ${ }^{74}$.

Fusion inhibition assay with split luciferase. The quantitative fusion inhibition assay was based on a dual-split-reporter described previously ${ }^{35,36,75}$. Receptor negative $\mathrm{CHO} 745$ cells $\left(7 \times 10^{5}\right.$ in a six-well plate) were transfected with $0.75 \mu \mathrm{g}$ each of the indicated HNV F and G glycoprotein expression plasmids and $0.5 \mu \mathrm{g}$ of an expression plasmid for one half of a split-luciferase-reporter protein (DSP1-7, a kind gift of Z. Matsuda). GeneJuice reagent (Millipore Sigma) was used for transfections according to the manufacturer's instructions. Concurrently, CHO745-ephrin-B2 cells $\left(1 \times 10^{4}\right.$ cells in a clear-bottom, black wall 96-well plate) were transfected with $100 \mathrm{ng}$ of the expression plasmid for other half of the split-luciferase-reporter protein (DSP8-11). Then, $24 \mathrm{~h}$ posttransfection, Versene (0.48 mM EDTA in PBS) (ThermoFisher Scientific) was used to gently detach the $\mathrm{CHO} 745$ cells from the six-well plate and $2.5 \times 10^{4}$ cells were incubated for $15 \mathrm{~min}$ at room temperature with the indicated $\mathrm{mAb}$ dilutions before being over-laid on the receptor-expressing CHO745-ephrin-B2 cells in the 96-well plate. Single $\mathrm{mAbs}(1 \mathrm{~F} 5,5 \mathrm{~B} 3$ and $12 \mathrm{~B} 2)$ were used at $100,10,1$ and $0.1 \mathrm{ng} \mathrm{ml}^{-1}$. Cocktail mAbs $(12 \mathrm{~B} 2 / 1 \mathrm{~F} 5$ and $12 \mathrm{~B} 2-5 \mathrm{~B} 3)$ were used at $50,5,0.5,0.25$ and $0.125 \mathrm{ng} \mathrm{ml}^{-1}$ of each $\mathrm{mAb}$. Negative control mAb D40 (HIV-gp-41-specific mAb) was tested at 100 and

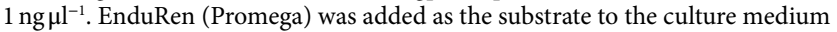
(DMEM, 10\%. FBS) according to the manufacturer's instructions. Reconstitution of luciferase function as a result of content mixing driven by cell-cell fusion between the glycoprotein expressing $\mathrm{CHO} 745$ cells and the receptor-bearing CHO745-ephrin-B2 cells was measured using an Infinite M200 Pro microplate reader (Tecan). Each data point comprises at least two technical repeats. The luciferase signal in the sample where there was no $\mathrm{mAb}$ added was used to define $100 \%$ fusion and calculate membrane fusion percentage for each $\mathrm{mAb}$ treatment.

Neutralization assays with authentic NiV-M, NiV-B or HeV virions. The virus infectivity neutralization concentrations of a control antibody c13G8 IgG specific to the GP38 protein of Crimean-Congo hemorrhagic fever virus $(\mathrm{CCHFV})^{76}$, murine 1F5 IgG, humanized h1F5 IgG, murine 12B2 IgG, humanized h12B2 IgG and antibodies cocktail (h12B2-h1F5, h12B2-h5B3.1) were determined for $\mathrm{NiV}-\mathrm{M}, \mathrm{NiV}-\mathrm{B}$ and $\mathrm{HeV}$ using a plaque reduction assay. Briefly, antibodies were serially diluted fivefold from $100 \mu \mathrm{g} \mathrm{ml}^{-1}$ to $6.4 \mathrm{ng} \mathrm{ml}^{-1}$ and incubated with a target of roughly 100 plaque-forming units (PFU) of NiV-M, NiV-B or $\mathrm{HeV}$ for $45 \mathrm{~min}$ at $37^{\circ} \mathrm{C}$. In the case of antibody cocktails, each $\mathrm{mAb}$ component in the cocktail was present at half of the indicated concentrations. The control mAb c13G8 was tested only at the highest concentration $\left(100 \mu \mathrm{g} \mathrm{ml}^{-1}\right)$. Virus and antibody mixtures were then added to individual wells of six-well plates of VeroE6 cells. Plates were stained with neutral red $2 \mathrm{~d}$ after infection and plaques were counted $24 \mathrm{~h}$ after staining. Neutralization potency was calculated based on PFU for each virus in the well without antibody. The experiments were performed in duplicate with independent virus preparations and duplicate readings for each replicate. $\mathrm{IC}_{50}$ values were calculated as previously described ${ }^{77}$. Kruskal-Wallis tests were used in Prism to compare groups of $\mathrm{IC}_{50}$ values determined from each replicate experiment for single mAbs versus $\mathrm{mAb}$ cocktails to assess whether they were statistically different

Analysis of viral escape mutants in the presence of mAbs. Authentic NiV-M was passaged three times in the presence of $100 \mu \mathrm{g}$ of antibody at each passage. The first passage was in the presence of 50,000 PFU of NiV-M and the additional passages were against 5,000 PFU (if possible). During each passage, the virus was incubated with $100 \mu \mathrm{g}$ of each antibody in $100 \mu \mathrm{l}$ total volume for $1 \mathrm{~h}$ at $37^{\circ} \mathrm{C}$ with rocking and flicking every $15 \mathrm{~min}$. The total volume was raised to $200 \mu \mathrm{l}$ by addition of $10 \%$ FBS in DMEM and incubated on Vero 76 cells in a $35 \mathrm{~mm}$ well, rocking every 15 minutes for $1 \mathrm{~h}$ at $37^{\circ} \mathrm{C}$. After the inoculum was removed, 10\% FBS in DMEM containing $100 \mu \mathrm{g}$ of $\mathrm{F} \mathrm{mAb}$ in the final inoculum and supernatants were collected 72 hours post-infection (hpi).

Supernatant from each passage was titered by plaque assay with Vero 76 cells. Briefly, increasing tenfold dilutions of the samples were adsorbed to Vero cell monolayers in duplicate wells $(200 \mu \mathrm{l})$; the limit of detection was $25 \mathrm{pfu} \mathrm{ml}^{-1}$.

Virus from the third passage was diluted to roughly $100 \mathrm{PFU}$ and incubated with $100 \mu \mathrm{g}$ of each $\mathrm{F} \mathrm{mAb}$ in $100 \mu \mathrm{l}$ of total volume for $1 \mathrm{~h}$ at $37^{\circ} \mathrm{C}$ with rocking/ flicking every $15 \mathrm{~min}$. The volume of the virus-F mAb mixture was then raised to
$200 \mu \mathrm{l}$ by addition of $10 \%$ FBS DMEM and incubated on Vero 76 cells in a $35 \mathrm{~mm}$ well, rocking every $15 \mathrm{~min}$ for $1 \mathrm{~h}$ at $37^{\circ} \mathrm{C}$. Cells were then overlayed with $0.9 \%$ agarose Eagle's Minimum Essential Medium (EMEM) and observed for plaque formation 24 to $48 \mathrm{~h}$ postoverlay. Five plaques per $\mathrm{F} \mathrm{mAb}$ and per virus were picked using a P1000 pipette tip and placed into $500 \mu \mathrm{l}$ of $10 \%$ FBS DMEM and allowed to diffuse for $10 \mathrm{~min}$ at room temperature and then placed at $-80^{\circ} \mathrm{C}$.

Plaque purified virus preparations were used to inoculate Vero 76 cells by rocking every $15 \mathrm{~min}$ for $1 \mathrm{~h}$ at $37^{\circ} \mathrm{C}$. After the inoculum was removed, $10 \%$ FBS DMEM was added and supernatants were collected $72 \mathrm{hpi}$ and $200 \mu \mathrm{l}$ of supernatants were placed into $1 \mathrm{ml}$ of Trizol LS for RNA extraction following the manufacturer's instructions (Life Technologies). RNA was reconstituted into $30 \mu \mathrm{l}$ of nuclease-free $\mathrm{dH}_{2} \mathrm{O}$

Negative staining EM analysis of NiV F-12B2-1F5 and NiV F-12B2-5B3 complexes. S-peptide tagged NiV F ectodomain was incubated overnight at $4{ }^{\circ} \mathrm{C}$ at with 12B2-1F5 Fabs or 12B2-5B3 Fabs at a molar ratio of 1:6:3 NiV F:12B2:1F5 or NiV F:12B2:5B3. Each complex was diluted to a concentration of $0.01 \mathrm{mg} \mathrm{ml}^{-1}$ NiV F immediately before absorption onto glow-discharged carbon-coated copper grids for $20 \mathrm{~s}$, followed by $2 \times$ application of $2 \%$ uranyl formate stain. Micrographs were recorded using Leginon software ${ }^{78}$ on a $120 \mathrm{kV}$ FEI Tecnai G2 Spirit with a Gatan Ultrascan $40004,000 \times 4,000$ CCD camera at 670,000 nominal magnification at a defocus range of $1.5-2.5 \mu \mathrm{m}$ and pixel size of $1.6 \AA$. Particles were picked using DoGPicker, a reference-free picker ${ }^{79}$. Contrast-transfer function was estimated using $\mathrm{GCTF}^{57}$. 2D class averages were generated in cryoSPARC ${ }^{59}$. Reference-free $\mathrm{ab}$ initio $3 \mathrm{D}$ reconstruction of selected particles from $2 \mathrm{D}$ class averages from each dataset was performed in cryoSPARC with eight classes. The $3 \mathrm{D}$ reconstructed class with the highest stoichiometry of Fabs bound are colored and shown in Supplementary Fig. 4a.

Reporting Summary. Further information on research design is available in the Nature Research Reporting Summary linked to this article.

\section{Data availability}

The cryo-EM maps and atomic models have been deposited to the Electron Microscopy Data Bank and Protein Data Bank with accession numbers EMD22884/PDB 7KI4 (NiV F-12B2) and EMD-22885/PDB 7KI6 (HeV F-1F5). Sharing of reagents will proceed through a Materials Transfer Agreement.

\section{References}

53. Huynh-Do, U. et al. Ephrin-B1 transduces signals to activate integrin-mediated migration, attachment and angiogenesis. J. Cell Sci. 115, 3073-3081 (2002)

54. Suloway, C. et al. Automated molecular microscopy: the new Leginon system. J. Struct. Biol. 151, 41-60 (2005).

55. Zheng, S. Q. et al. MotionCor2: anisotropic correction of beam-induced motion for improved cryo-electron microscopy. Nat. Methods 14, 331-332 (2017)

56. Lander, G. C. et al. Appion: an integrated, database-driven pipeline to facilitate EM image processing. J. Struct. Biol. 166, 95-102 (2009).

57. Zhang, K. GCTF: real-time CTF determination and correction. J. Struct. Biol. 193, 1-12 (2016).

58. Zivanov, J. et al. New tools for automated high-resolution cryo-EM structure determination in RELION-3. Elife 7, e42166 (2018).

59. Punjani, A., Rubinstein, J. L., Fleet, D. J. \& Brubaker, M. A. CryoSPARC: algorithms for rapid unsupervised cryo-EM structure determination. Nat. Methods 14, 290-296 (2017).

60. Zivanov, J., Nakane, T. \& Scheres, S. H. W. A Bayesian approach to beam-induced motion correction in cryo-EM single-particle analysis. IUCrJ 6, 5-17 (2019)

61. Punjani, A., Zhang, H. \& Fleet, D. J. Non-uniform refinement: adaptive regularization improves single-particle cryo-EM reconstruction. Nat. Methods 17, 1214-1221 (2020).

62. Scheres, S. H. W. \& Chen, S. Prevention of overfitting in cryo-EM structure determination. Nat. Methods 9, 853-854 (2012).

63. Rosenthal, P. B. \& Henderson, R. Optimal determination of particle orientation, absolute hand, and contrast loss in single-particle electron cryomicroscopy. J. Mol. Biol. 333, 721-745 (2003).

64. Chen, S. et al. High-resolution noise substitution to measure overfitting and validate resolution in $3 \mathrm{D}$ structure determination by single particle electron cryomicroscopy. Ultramicroscopy 135, 24-35 (2013).

65. Goddard, T. D., Huang, C. C. \& Ferrin, T. E. Visualizing density maps with UCSF Chimera. J. Struct. Biol. 157, 281-287 (2007).

66. Brown, A. et al. Tools for macromolecular model building and refinement into electron cryo-microscopy reconstructions. Acta Crystallogr. D Biol. Crystallogr. 71, 136-153 (2015).

67. Dimaio, F. et al. Atomic-accuracy models from $4.5-\AA$ cryo-electron microscopy data with density-guided iterative local refinement. Nat. Methods 12, 361-365 (2015). 
68. DiMaio, F., Leaver-Fay, A., Bradley, P., Baker, D. \& André, I. Modeling symmetric macromolecular structures in Rosetta3. PLoS ONE 6, e20450 (2011).

69. Wang, R. Y. R. et al. Automated structure refinement of macromolecular assemblies from cryo-EM maps using Rosetta. Elife 5, e17219 (2016).

70. Frenz, B. et al. Automatically fixing errors in glycoprotein structures with Rosetta. Structure 27, 134-139.e3 (2019).

71. Chen, V. B. et al. MolProbity: all-atom structure validation for macromolecular crystallography. Acta Crystallogr. D Biol. Crystallogr. 66, 12-21 (2010).

72. Barad, B. A. et al. EMRinger: side chain-directed model and map validation for 3D cryo-electron microscopy. Nat. Methods 12, 943-946 (2015).

73. Agirre, J. et al. Privateer: software for the conformational validation of carbohydrate structures. Nat. Struct. Mol. Biol. 22, 833-834 (2015).

74. Goddard, T. D. et al. UCSF ChimeraX: meeting modern challenges in visualization and analysis. Protein Sci. 27, 14-25 (2018).

75. Laing, E. D. et al. Rescue and characterization of recombinant cedar virus, a non-pathogenic Henipavirus species. Virol. J. 15, 56 (2018).

76. Mishra, A. K. et al. Structure and characterization of Crimean-Congo hemorrhagic fever virus GP38. J. Virol. https://doi.org/10.1128/jvi.02005-19 (2020).

77. Ferrara, F. \& Temperton, N. Pseudotype neutralization assays: from laboratory bench to data analysis. Methods Protoc. 1, 8 (2018).

78. Suloway, C. et al. Automated molecular microscopy: the new Leginon system. J. Struct. Biol. 151, 41-60 (2005).

79. Voss, N. R., Yoshioka, C. K., Radermacher, M., Potter, C. S. \& Carragher, B. DoG Picker and TiltPicker: software tools to facilitate particle selection in single particle electron microscopy. J. Struct. Biol. 166, 205-213 (2009).

\section{Acknowledgements}

This study was supported by the National Institute of Allergy and Infectious Diseases (grant nos. DP1AI158186 and HHSN272201700059C to D.V. and grant nos. AI054715, AI077995 and AI142764 to C.C.B.), the National Institute of General Medical Sciences (grant no. GM120553 to D.V.), an Investigators in the Pathogenesis of Infectious Disease Award from the Burroughs Wellcome Fund (D.V.), a Pew Biomedical Scholars Award (D.V.) and the University of Washington Arnold and Mabel Beckman cryo-EM center.
Operations support of the Galveston National Laboratory was supported by NIAID/NIH grant no. UC7AI094660.

\section{Author contributions}

H.V.D., R.W.C., T.W.G., C.C.B. and D.V. designed the experiments. A.S.D., L.Y. and Y.-P.C. designed and cloned the $\mathrm{HeV} F$ and NiV F constructs and produced and isolated 1F5, 5B3 and 12B2 mouse hybridoma and mAbs. H.V.D. expressed and purified the soluble HNV F proteins used in this study. B.R.W., L.Z. and Z.A.B. performed humanization of murine mAbs and produced the humanized mAbs. H.V.D. performed IgG fragmentation and binding assays. H.V.D. conducted the cryo-EM sample preparation, data collection and data processing. H.V.D. and D.V. built and refined the atomic models. R.W.C. carried out the neutralization assays. V.B., C.M. and T.W.G. performed escape mutant analyses. C.K.N. carried out the membrane fusion inhibition assay. M.A. prepared a stable cell line. H.V.D., R.W.C., S.C.D.S., C.C.B. and D.V. analyzed the data. H.V.D. and D.V. prepared the manuscript with input from all authors.

\section{Competing interests}

Z.A.B., B.R.W. and L.Z. are employees and shareholders in Mapp Biopharmaceutical Inc., and L.Z. is a co-owner of Mapp Biopharmaceutical, Inc. D.V. is a consultant for Vir Biotechnology Inc. The Veesler laboratory has received an unrelated sponsored research agreement from Vir Biotechnology Inc. The other authors declare no competing interests.

\section{Additional information}

Extended data is available for this paper at https://doi.org/10.1038/s41594-021-00584-8.

Supplementary information The online version contains supplementary material available at https://doi.org/10.1038/s41594-021-00584-8.

Correspondence and requests for materials should be addressed to D.V.

Peer review information Nature Structural \& Molecular Biology thanks Dimiter Dimitrov and the other, anonymous, reviewer(s) for their contribution to the peer review of this work. Peer reviewer reports are available. Beth Moorefield was the primary editor on this article and managed its editorial process and peer review in collaboration with the rest of the editorial team.

Reprints and permissions information is available at www.nature.com/reprints. 
a

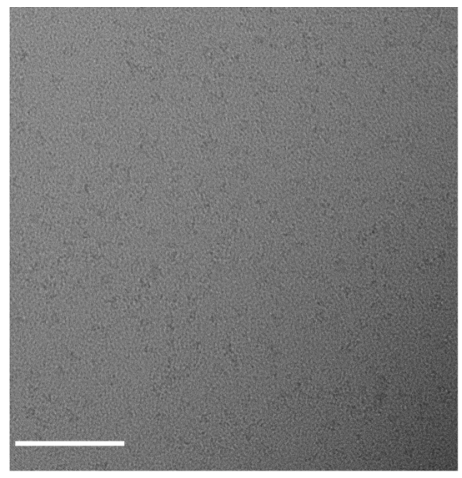

b

\section{\& $* 4<4$}

d

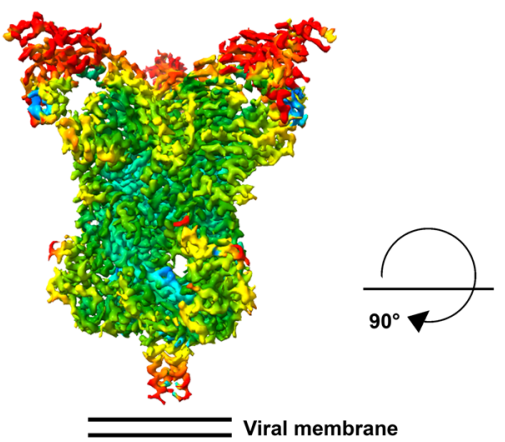

e

\section{2,113 movies \\ MotionCor2 \\ GCTF \\ DogPicker \\ $\checkmark x$ traction}

cryosPARC
C
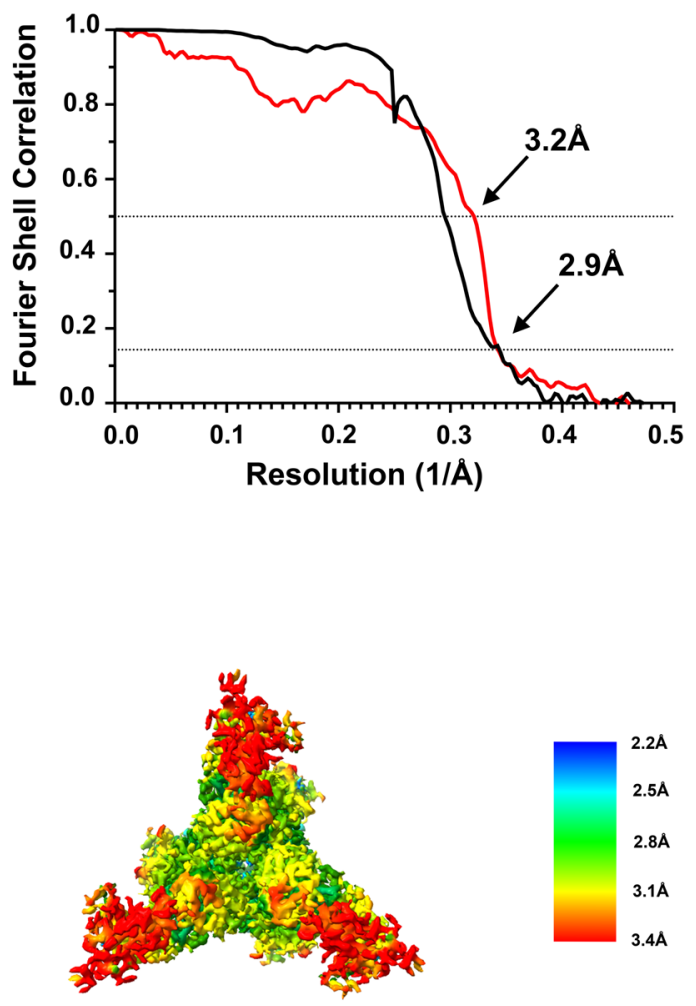

cryoSPARC

Ab initio (C1) 41,574

NUR (C3)

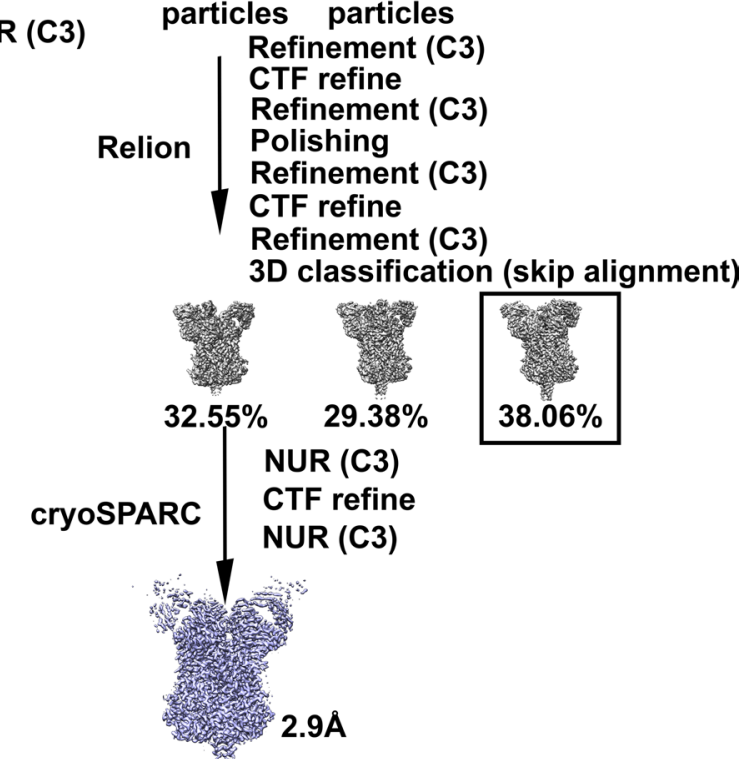

Extended Data Fig. 1 | See next page for caption. 
Extended Data Fig. 1 | Cryo-EM characterization of NiV F in complex with the 12B2 Fab fragment. a, Representative micrograph. Scale bar, $100 \mathrm{~nm}$.

b, Reference-free 2D class averages. Scale bar, $100 \AA$. c, Gold-standard (black) and map-model (red) Fourier shell correlation curves. Dotted line indicates the 0.143 and 0.5 thresholds. d, Two orthogonal views of the cryo-EM map colored by local resolution estimated using cryoSPARC. e, Cryo-EM data processing flow chart. Selected groups of particles at different steps are boxed. NUR: Non-Uniform refinement. 
12B2 binding to NiV F S69A

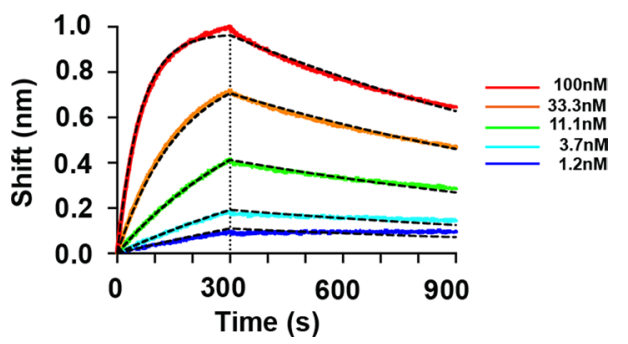

Extended Data Fig. 2 | Binding of the 12B2 Fab fragment to immobilized NiV F S69A ectodomain (N67 glycan mutant) analyzed by biolayer interferometry. Raw data are colored according to the key and fitted curves are displayed as black dashed lines. The vertical dotted lines correspond to the transition between the association and dissociation phases. 
a

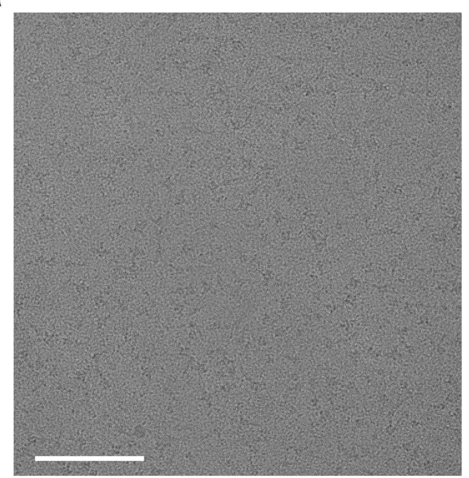

b

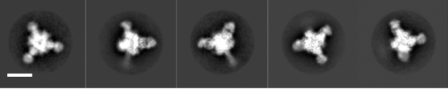

C

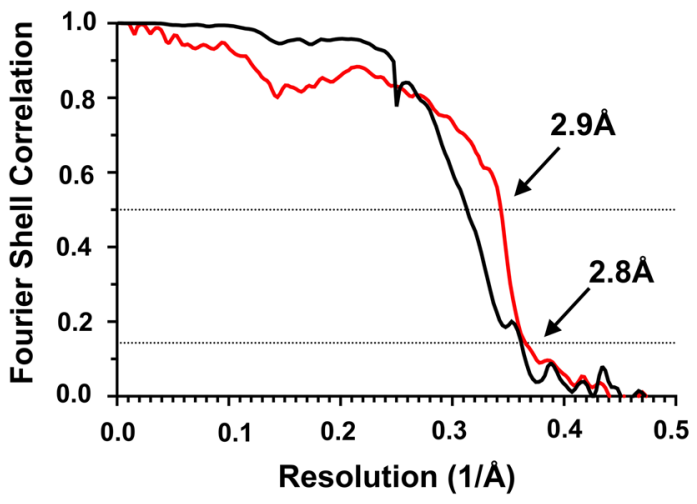

d
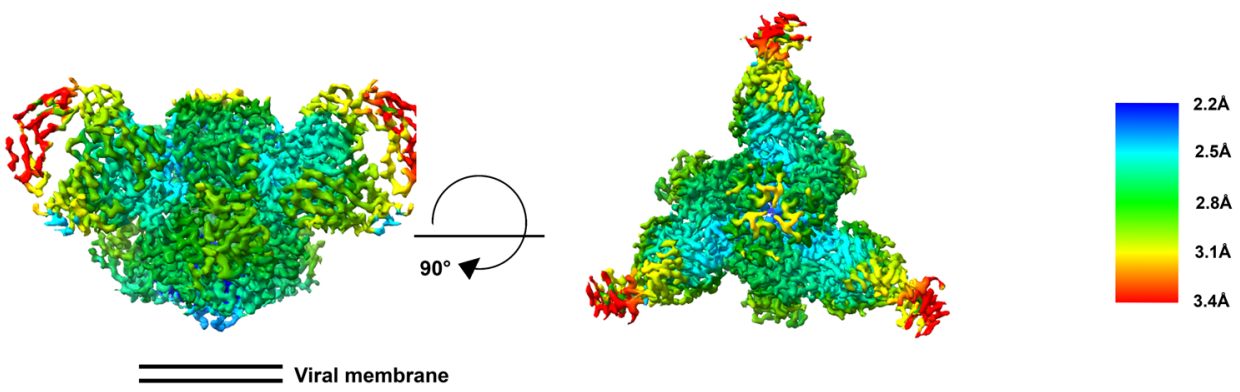

e

1,794 movies

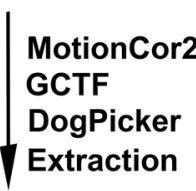

432,222 particles Relion 2D classification 99,247 particles

Relion

3D classification (C1)
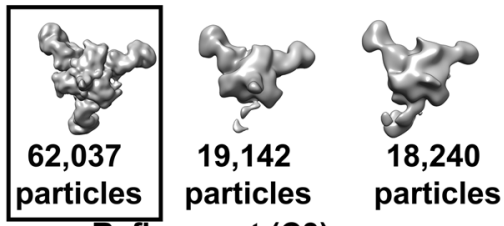

Refinement (C3)

CTF refine

Refinement (C3)

Relion

Polishing

Refinement (C3)

CTF refine

Refinement (C3)

3D classification (skip alignment)

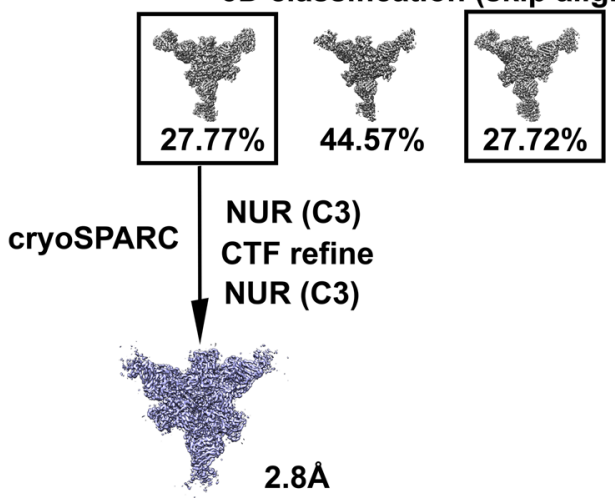


Extended Data Fig. 3 | CryoEM characterization of HeV F in complex with the 1F5 Fab fragment. a, Representative micrograph. Scale bar, $100 \mathrm{~nm}$.

b, Reference-free 2D class averages. Scale bar, $100 \AA$ A. c, Gold-standard (black) and map-model (red) Fourier shell correlation curves. Dotted line indicates the 0.143 and 0.5 thresholds. d, Two orthogonal views of the cryo-EM map colored by local resolution estimated using cryoSPARC. e, Cryo-EM data processing flow chart. Selected groups of particles at different steps are boxed. NUR: Non-Uniform refinement. 
a

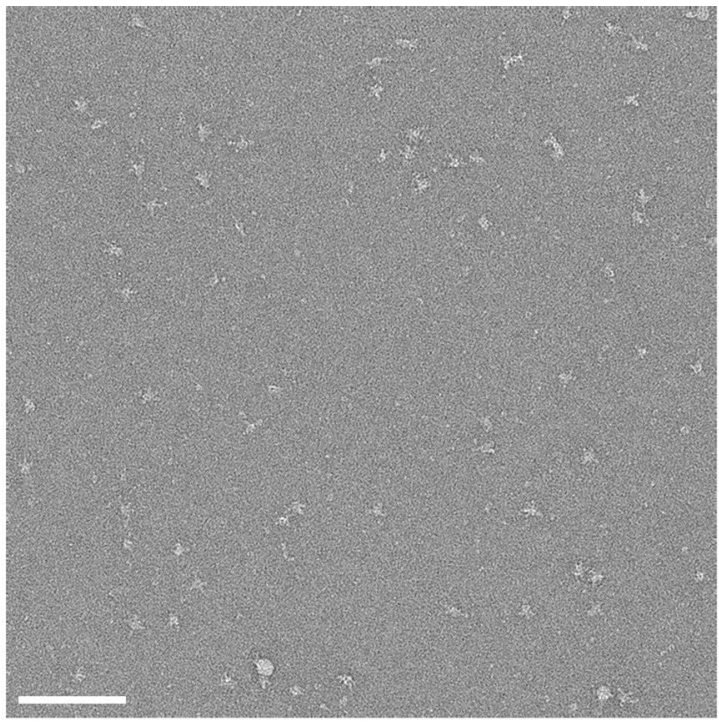

b

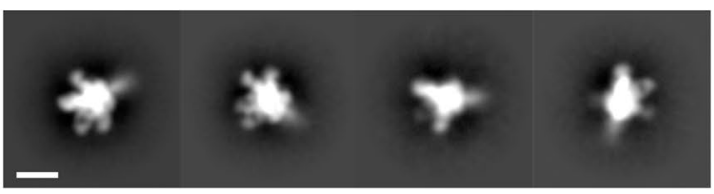

d

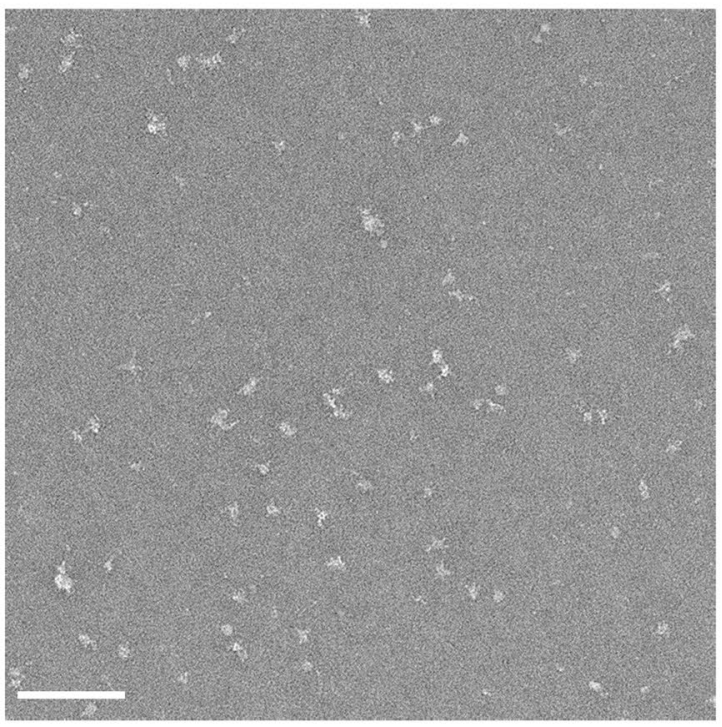

e

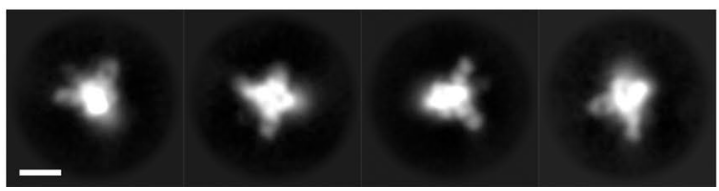

C

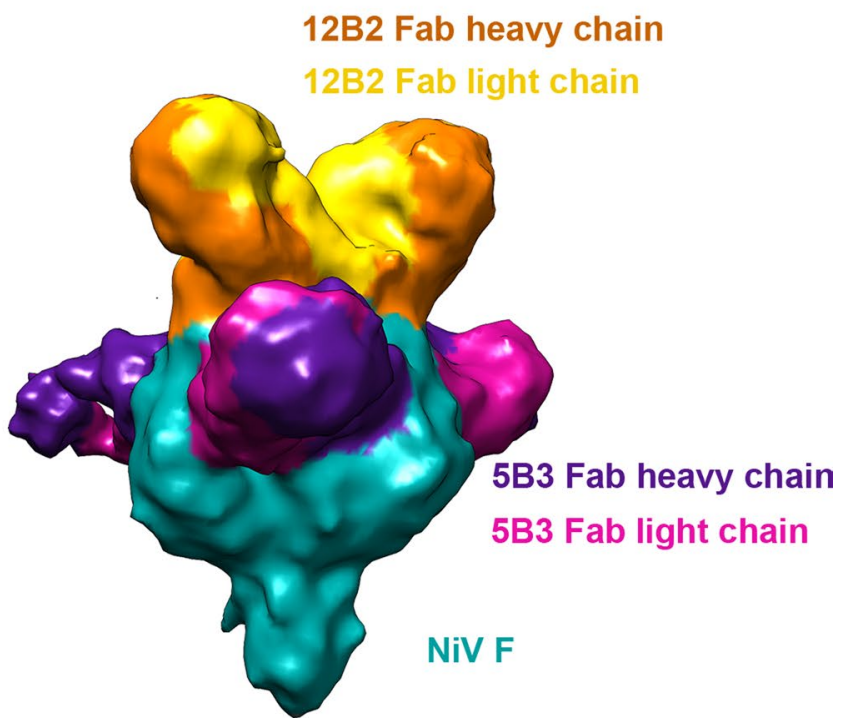

Viral membrane

f

12B2 Fab heavy chain

12B2 Fab light chain

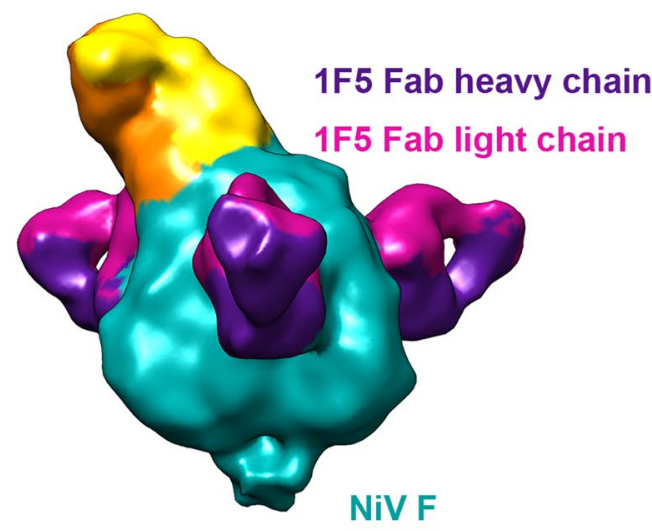

\section{Viral membrane}

Extended Data Fig. 4 | EM characterization of the negatively stained ternary complex of NiV F/12B2/5B3 and NiV F/12B2/1F5. a,c, A representative micrograph and 2D class averages of NiV F/12B2/5B3 complex (a) and NiV F/12B2/1F5 complex (c) from the corresponding negative staining dataset of NiV F incubated with excess of 12B2/1F5 Fabs or 12B2/5B3 Fabs (See Materials \& Methods). Micrograph scale bar: 100 nm; 2D class average scale bar: $100 \AA . \mathbf{b}, \mathbf{d}$, Three-dimensional reconstructions of the ternary complex of NiV F/12B2/5B3 (b) and NiV F/12B2/1F5 (d), representing a complex with the highest stoichiometry of Fabs:NiV F from the corresponding negative staining dataset. 


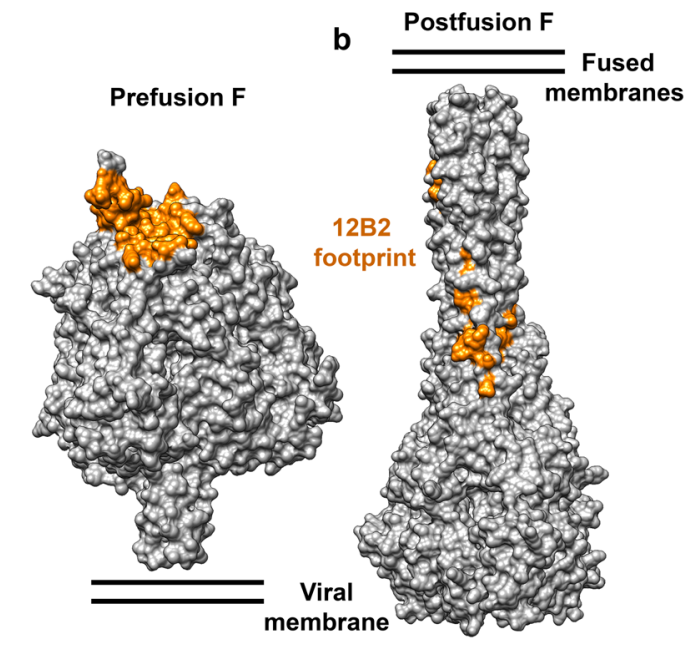

C

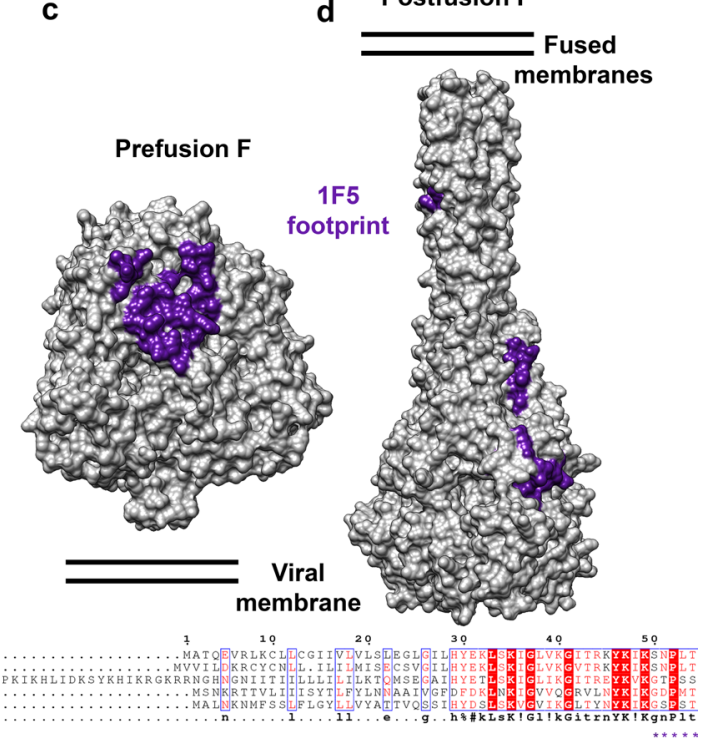

e
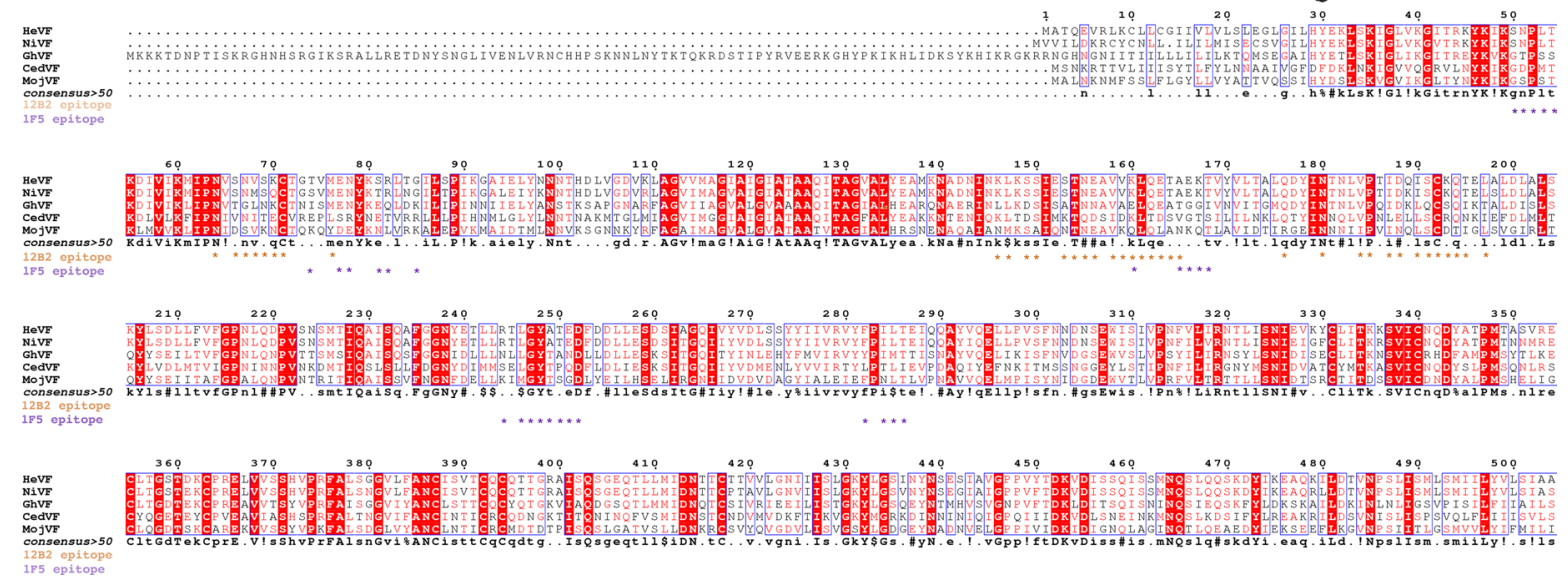

Extended Data Fig. 5 | Comparison of the footprints of the 12B2 and the 1F5 antibody on prefusion $F$ and postfusion $F$ and among HNV F proteins. a, $b$, Molecular surface representation of the NiV F prefusion trimer (a) and the homology model of NiV F postfusion (b) showing the 12B2 footprint in orange. c-d, Molecular surface representation of the HeV F prefusion trimer (c) and the homology model of NiV F postfusion (d) showing the 1F5 footprint in purple. The homology model of NiV F postfusion in (b) and (d) was obtained by threading the NiV F sequence onto the human parainfluenza postfusion F structure ${ }^{23}$ (PDB: 1ZTM). e, Sequence alignment of HNV F glycoproteins (NiV, HeV, GhV: Ghana bat virus; CedV: Cedar virus; MojVF: Mojiang virus). Residues on HNV F constituting the 12B2 or 1F5 epitope are denoted with an orange or purple asterisk, respectively. 


\section{Reporting Summary}

Nature Research wishes to improve the reproducibility of the work that we publish. This form provides structure for consistency and transparency in reporting. For further information on Nature Research policies, see our Editorial Policies and the Editorial Policy Checklist.

\section{Statistics}

For all statistical analyses, confirm that the following items are present in the figure legend, table legend, main text, or Methods section.

$\mathrm{n} / \mathrm{a}$ Confirmed

$\bigotimes$ The exact sample size $(n)$ for each experimental group/condition, given as a discrete number and unit of measurement

\ A statement on whether measurements were taken from distinct samples or whether the same sample was measured repeatedly

$\triangle$ The statistical test(s) used AND whether they are one- or two-sided

Only common tests should be described solely by name; describe more complex techniques in the Methods section.

Х $\square$ A description of all covariates tested

Х $\square$ A description of any assumptions or corrections, such as tests of normality and adjustment for multiple comparisons

A full description of the statistical parameters including central tendency (e.g. means) or other basic estimates (e.g. regression coefficient)

$X$ AND variation (e.g. standard deviation) or associated estimates of uncertainty (e.g. confidence intervals)

$X$ For null hypothesis testing, the test statistic (e.g. $F, t, r$ ) with confidence intervals, effect sizes, degrees of freedom and $P$ value noted

Х Give $P$ values as exact values whenever suitable.

Х $\square$ For Bayesian analysis, information on the choice of priors and Markov chain Monte Carlo settings

Х $\square$ For hierarchical and complex designs, identification of the appropriate level for tests and full reporting of outcomes

Х $\square$ Estimates of effect sizes (e.g. Cohen's $d$, Pearson's $r$ ), indicating how they were calculated

Our web collection on statistics for biologists contains articles on many of the points above.

\section{Software and code}

Policy information about availability of computer code

Data collection N/A

Data analysis N/A

For manuscripts utilizing custom algorithms or software that are central to the research but not yet described in published literature, software must be made available to editors and

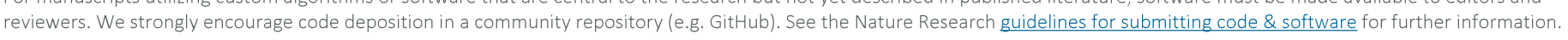

\section{Data}

Policy information about availability of data

All manuscripts must include a data availability statement. This statement should provide the following information, where applicable:

- Accession codes, unique identifiers, or web links for publicly available datasets

- A list of figures that have associated raw data

- A description of any restrictions on data availability

HeV F-1F5 (EMD- 22885, PDB: 7KI6)

NiV F-12B2 (EMD- 22884, PDB: 7KI4) 


\section{Field-specific reporting}

Please select the one below that is the best fit for your research. If you are not sure, read the appropriate sections before making your selection.

$\bigotimes$ Life sciences

Behavioural \& social sciences

Ecological, evolutionary \& environmental sciences

For a reference copy of the document with all sections, see nature.com/documents/nr-reporting-summary-flat.pdf

\section{Life sciences study design}

All studies must disclose on these points even when the disclosure is negative.

Sample size N/A
Data exclusions N/A
Replication N/A
Randomization N/A

\section{Reporting for specific materials, systems and methods}

We require information from authors about some types of materials, experimental systems and methods used in many studies. Here, indicate whether each material, system or method listed is relevant to your study. If you are not sure if a list item applies to your research, read the appropriate section before selecting a response.

\begin{tabular}{l|l} 
Materials \& experimental system \\
\hline $\mathrm{n} / \mathrm{a}$ & Involved in the study \\
\hline & $\bigotimes$ Antibodies \\
$\square$ & $\bigotimes$ Eukaryotic cell lines \\
$\square$ & $\square$ Palaeontology and archaeology \\
$\square$ & $\square$ Animals and other organisms \\
$\searrow$ & $\square$ Clinical data \\
$\searrow$ & $\square$ Dual use research of concern
\end{tabular}

\begin{tabular}{l|l}
\multicolumn{2}{l}{ Methods } \\
\hline n/a & Involved in the study \\
$\bigotimes$ & $\square$ ChIP-seq \\
$\searrow$ & $\square$ Flow cytometry \\
$\bigotimes$ & $\square$ MRI-based neuroimaging
\end{tabular}

Antibodies

Antibodies used $\quad$ IF5 and 12B2 discovered in this study and sequences will be released along with the PDB coordinates
Validation

\section{Eukaryotic cell lines}

Policy information about cell lines

Cell line source(s)

HEK293F, CHO

Authentication

None of the cell lines used were authenticated

Mycoplasma contamination

Not tested

Commonly misidentified lines

(See ICLAC register)

N/A 